\title{
Compliance Strategies under Permits for Emissions
}

\author{
Ravi Subramanian • Sudheer Gupta • Brian Talbot \\ College of Management, Georgia Institute of Technology, 800 West Peachtree Street NW, \\ Atlanta, Georgia 30332, USA \\ Faculty of Business Administration, Simon Fraser University, 8888 University Drive, Burnaby, \\ British Columbia V5A 1S6, Canada \\ Stephen M. Ross School of Business, University of Michigan, 701 Tappan Street, Ann Arbor, Michigan 48109, USA \\ ravi.subramanian@mgt.gatech.edu • sudheerg@sfu.ca・btalbot@umich.edu
}

\begin{abstract}
$\mathrm{W}$ e characterize the trade-offs among firms' compliance strategies in a market-based program where a regulator interested in controlling emissions from a given set of sources auctions off a fixed number of emissions permits. We model a three-stage game in which firms invest in emissions abatement, participate in a share auction for permits, and produce output. We develop a methodology for a profit-maximizing firm to derive its marginal value function for permits and translate this value function into an optimal bidding strategy in the auction. We analyze two end-product market scenarios-independent demands and Cournot competition. In both scenarios we find that changing the number of available permits influences abatement to a lesser extent in a dirty industry than in a cleaner one. In addition, abatement levels taper off with increasing industry dirtiness levels. In the presence of competition, firms in a relatively clean industry can, in fact, benefit from a reduction in the number of available permits. Our findings are robust to changes in certain modeling assumptions.
\end{abstract}

Key words: environmental compliance; pollution permits; market-based mechanisms; auctions; game theory

Submissions and Acceptance: Received June 2005; revisions received June 2006 and November; accepted January 2007 by Luk van Wassenhove.

\section{Introduction}

Market-based policy instruments are now being considered for a range of environmental issues, from endangered species preservation to the greenhouse effect and global climate change (The Wall Street Journal 2006, 2005a,b,c; Stavins 1998). Emissions trading programs for pollutants such as lead, sulfur dioxide $\left(\mathrm{SO}_{2}\right)$, nitrogen oxides $\left(\mathrm{NO}_{\mathrm{x}}\right)$, carbon dioxide $\left(\mathrm{CO}_{2}\right)$, and volatile organic compounds (VOCs), are prime examples. Notably, the EU greenhouse gas Emissions Trading Scheme (ETS), which came into force in 2005, caps $\mathrm{CO}_{2}$ emissions from more than 12,000 industrial plants and is a precursor to similar systems that will be implemented under the Kyoto Protocol. Emissions trading refers to a market-based mechanism that allows parties to buy and sell permits to emit certain pollutants. It differs from the traditional command-and-control approach that relies on an agency, usually the government, to issue standards and specific directives on the amounts by which firms must reduce their emissions, how they must do so, and the penalties for failure (NRTEE 2002). The total number of permits issued corresponds to the overall emissions target of the covered sources. The fact that the target is less than "business as usual" emissions creates permit scarcity, resulting in a market price for permits. The concept of emissions trading has evolved into a central idea in environmental regulation, with well-developed theory [see Tietenberg (2001) for a comprehensive collection of articles on emissions trading], and attention has now shifted to how trading programs should be implemented (Muller and Mestelman 1998).

Most research to date has focused on evaluating the efficacy of emissions trading programs vis- $a$-vis other approaches such as taxes, subsidies, and standards from a regulator's perspective. We instead take the perspective of a profit-maximizing firm and focus on the trade-offs among different compliance strategies 
that a firm has at its disposal. We consider three such strategies-investment in abatement, procurement of permits, and adjustment of output levels-in a program based on auctioned permits. The total number of permits available to firms is exogenous to our model and is decided by the regulator. To the regulator, knowledge about the interrelationships among firm levers for compliance is crucial given the goals of pollution control and a desired increasing level of stringency in pollution limits. From a firm's perspective, decisions such as investment in pollution abatement, permit bidding strategy, and production level must be made given policy stipulations, the accompanying cost of compliance, and the goal of profit maximization.

In practice, permits are allocated by "grandfathering" or by auctioning. Grandfathering refers to the process of allocating permits to firms free of charge, based on historical emissions or process inputs. Free allocation is problematic in terms of efficiency because it conveys scarcity rents to the polluting firms (Fullerton and Metcalf 2001). Grandfathered permits also give rise to entry barriers, because new entrants must purchase permits from existing holders (Stavins 1998). Cramton and Kerr (2002) argue that auctioning is superior to grandfathering because it is much more efficient, allows reduced tax distortions, provides greater incentives for innovation, provides more flexibility in distribution of costs, and reduces the need for politically contentious arguments over the allocation of rents. Moreover, auctioning serves to increase liquidity in permit markets and to facilitate the permit price discovery process (Schmalensee et al. 1998). Despite the apparent economic superiority of auctions over free distribution, the latter is implemented because it offers a greater degree of political control over the distributional effects of regulation (Stavins 1998). We focus on auctioning as the primary means for allocating permits but we do provide a brief discussion in Section 7.2 on how our approach can be extended to incorporate grandfathering in addition to auctioning.

Permit auctions are implemented in various formats. The US EPA auctions for $\mathrm{SO}_{2}$ permits are in the sealed-bid discriminatory price format, and the Chicago Climate Exchange (CCX) auctions for $\mathrm{CO}_{2}$ permits are held in two formats-sealed-bid average price and sealed-bid discriminatory price. We model a sealed-bid uniform price "share" auction where the bidders submit a schedule of prices for varying fractions of the block of permits being auctioned and receive permits at a price that equates demand and supply. Although permits are sold in discrete units, modeling a share or divisible-good auction is appropriate because permits are homogeneous and the total number of auctioned permits is generally large
(125,000-150,000 in US EPA auctions of $\mathrm{SO}_{2}$ permits). It is worth mentioning that analytical results on equilibria in divisible good auctions are scarce (Hortaçsu 2002). However, there has been substantial debate on whether the discriminatory price format or the uniform price format is superior (Back and Zender 1993; Bikhchandani and Huang 1993; Daripa 2001). Our choice of a uniform price auction is driven by reasons of analytical tractability. Cramton and Kerr (2002) argue that when no bidder has significant market power, uniform pricing is nearly as efficient as Vickrey pricing and that, among sealed-bid auctions, a uniform price auction is probably the best. Despite the discriminatory price format of auctions for $\mathrm{SO}_{2}$ permits, prices have been in a narrow range. For example, in the EPA's March 2006 spot auction for $\mathrm{SO}_{2}$ permits, the difference between the average and lowest winning bids was $2.61 \%$. In any case, the modeling framework we employ is not limited to our auction format choice. As results become available in auction theory, they can be included within our framework.

We consider two scenarios of imperfect competition in the end-product market. In the first scenario, firms targeted by the regulator are local monopolies in the end-product market. An example is the US Acid Rain Program for restricting $\mathrm{SO}_{2}$ emissions from fossil fuelfired electric utilities. In the second scenario, firms compete for end-customer demand. An example is the EU emissions trading program for $\mathrm{CO}_{2}$, which includes major competitors in the steel industry such as ThyssenKrupp and Arcelor. In both demand scenarios, however, firms do compete for scarce emissions permits. We present a methodology by which a firm can derive its marginal value function for permits and translate this value function into an optimal bidding strategy in the auction for permits. In addition, the modeling effort provides a means for firms to deduce their optimal levels of abatement and output. We use the term "abatement" to refer to specific technological or process changes that result in lower emissions per unit of output (e.g., through the installation of scrubbers) or that result in a reduction in emissions independent of output (e.g., through the sequestration of exhaust gases). This is in contrast to some studies that refer to abatement as a black-box reduction in emissions from a baseline. In both demand scenarios we find that increasing stringency through a reduction in the total number of permits induces lower levels of abatement from firms in a "dirtier" industry than from those in a "cleaner" one (the level of dirtiness corresponds to the emissions per unit of output during production). In addition, abatement levels taper off with increasing industry dirtiness levels. In the presence of Cournot competition in the output market, firms in a relatively clean industry can, in fact, benefit from a reduction in the number of available permits, 
running counter to the common wisdom that an increasing stringency is detrimental to firm profitability [also see Bovenberg, Goulder, and Gurney (2004) and Smith, Ross, and Montgomery (2002) for related discussions on the conditions under which firm profits can be preserved under increasing stringency]. Our findings are robust to changes in certain modeling assumptions and help in understanding the trade-offs among abatement, permits, and output levels.

The remainder of this paper is organized as follows. Section 2 includes a review of the related literature. Section 3 describes the model. Sections 4 and 5, respectively, treat the two end-product market scenariosindependent demands and Cournot competition. Section 6 illustrates our results through a numerical example. Section 7 extends the analysis to: (i) a variant of the emissions function, (ii) the possibility that permits can be grandfathered in addition to being auctioned, (iii) the possibility that investment in abatement can affect the cost of production, and, (iv) firm heterogeneity. We discuss our results and provide directions for future research in Section 8. Proofs and tables of results are included in Appendices A and B, respectively.

\section{Literature}

To the best of our knowledge, ours is the first framework that enables an assessment of compliance strategies in a permit-based program for emissions control for varying policy stringency and firm dirtiness levels. Lyon (1986) examines equilibrium properties of a range of auctions and other procedures for allocating transferable permits. The paper's focus is on transferneutral (implying that no net revenue is generated for the seller) mechanisms that allocate permits efficiently, but production relationships or abatement are not modeled. Since 1995, the MIT Center for Energy and Environmental Policy Research (CEEPR) has published a series of empirical studies on the implementation of the Acid Rain Program in the US, including EPA auctions for $\mathrm{SO}_{2}$ permits. Laffont and Tirole (1996a) study the impact of spot and futures markets for tradable permits on firms' compliance decisions. Firms can buy permits, invest in abatement, or stop production and source out. The authors find that stand-alone spot markets induce excessive investment. The introduction of a futures market reduces this incentive to invest. Laffont and Tirole (1996b) extend this analysis assuming innovation is a public good and find that options to pollute at a given strike price fare better than permits from a social welfare point of view. However, they treat abatement investment as well as the choice between investment in abatement and production as binary decisions. In addition, they do not model permit auctions. Unold and
Requate (2001) propose a combination consisting of free permits and a menu of call options when there is imperfect information about aggregate abatement costs so that the regulator can approximate the marginal damage function. The authors, however, do not model permit auctions, nor do they explicitly model abatement and production relationships. Sunnevåg (2003) provides insights, based on numerical scenarios, into two competing permit auction designs-the standard ascending-clock auction and an alternative ascending-clock implementation of Vickrey pricingwhere the allocation of permits affects production and market shares. Montero (2002) compares R\&D incentives under four policy instruments-emission standards, performance standards, tradeable permits, and auctioned permits - with various strategic interactions in permit and output markets. Equilibrium permit price for the case of auctioned permits is established via a Nash bargaining argument in a two-player game-independent of the auction format. This is in contrast to our explicit derivation of the equilibrium bidding strategy and permit price based on the marginal value function for permits and on the auction format. Montero (2002) focuses on R\&D incentives in contrast to our characterization of the trade-offs among compliance levers. Bovenberg, Goulder, and Gurney (2004) and Smith, Ross, and Montgomery (2002) analyze the "equity-efficiency trade-off" in a general equilibrium model and focus on the fraction of permits that a regulator would want to allocate freely to firms to preserve industry profits. The regulator uses the revenue raised from permit auctions to lower distortionary taxes on factor inputs such as capital and labor. Our focus, on the other hand, is on understanding firm behavior under an exogenously specified emissions cap. Fischer, Parry, and Pizer (2003) model a three-stage process of innovation, diffusion, and emissions abatement in an oligopoly where firms are price takers. They compare the welfare effects of emissions taxes, auctioned permits, and grandfathered permits. One or many of the firms innovate and the rest are "adopters." Production relationships are not explicitly treated and the equilibrium permit price for the case of auctioned permits is exogenously specified. None of the above papers explicitly models investment in abatement, permit valuation and bidding, and output together with insights into equilibrium reactions of firms for varying stringency and dirtiness levels.

There is a growing stream of literature in operations management that studies the interface between operational decisions and the environment, at both the strategic and the tactical level [see Guide and Wassenhove (2006a,b); Kleindorfer, Singhal, and Van Wassenhove (2005); Corbett and Kleindorfer (2001a,b), and the papers referenced therein]. A range of topics has 
been studied in the context of closed-loop supply chains, including facility location, product and process design, inventory management, yield management, the value of information, contracts, and the impact of competition [e.g., Bakal and Akçali (2006); Ferguson and Toktay (2006); Debo, Toktay, and Van Wassenhove (2005); Savaskan, Bhattacharya, and Van Wassenhove (2003); Rajaram and Corbett (2002); and Stuart, Ammons, and Turbini (1999)]. We complement the aforesaid by analyzing compliance strategies for firms under explicit regulatory constraints during the process of production.

\section{Model}

We model a three-stage game in a symmetric oligopoly. In the first stage, each of the $n \geq 2$ firms decides its abatement level $\mu_{i}$ from an investment $\xi \mu_{i}^{2}$ in pollution mitigation (e.g., investments that enable the use of cleaner inputs or the scrubbing of flue gases). Quadratic abatement costs are commonly modeled in the literature [e.g., see Kennedy (2002) and Parry and Toman (2002)] and reflect diminishing returns to abatement investments. We model abatement as the first-stage decision since it is typically a long-term decision made at an early stage within the planning horizon. In programs such as the US EPA's $\mathrm{SO}_{2}$ permit program, a prespecified number of permits is auctioned off every year - a rate that is usually faster than the abatement investment cycle [also see Ellerman (1998)]. In the second stage of our model, firms bid for permits in a sealed-bid uniform price share auction conducted by the regulator. Note that if the abatement investment cycle is faster than the permit supply cycle, a sequence of decisions where the allocation of permits precedes the abatement decision might be more appropriate. However, analysis of such a sequence is intractable due to the complex permit valuation function that results (permit valuation is discussed in Section 4.2.1). Each firm submits a schedule of prices for varying fractional shares of the total available permits. The regulator then selects the market clearing price such that total demand equals the available supply. As in Wilson (1979), we assume that the number of bidders is known beforehand by all participants to be $n$. The auction results in a share allocation vector $s=\left(s_{1}\right.$, $\left.s_{2}, \ldots, s_{n}\right)$ or, equivalently, a permit allocation vector $\beta=\left(\beta_{1}, \beta_{2}, \ldots, \beta_{n}\right) . B=\sum_{i=1}^{n} \beta_{i}$ is the total permissible pollution level, where $\beta_{i}=s_{i} B$. The regulator's choice of the target $B$ is assumed to be exogenous to the model. Each permit allows a firm to emit one unit of the pollutant; $e$ is the permit price. In the third stage, each firm $i$ produces output $y_{i}$, which results in a pollution level $\left[\alpha /\left(1+\mu_{i}\right)\right] y_{i}$, and firms redeem their permits against their pollution levels. $\alpha$ is the emissions per unit of output and defines the cleanliness of firms or, equivalently, of technologies employed. The aforementioned functional form of the pollution level is representative of that in industries such as electric utilities [see Burtraw (2000) and Montero and Ellerman (1998)]. Section 7.1 treats the case where pollution reduction is independent of the output level. We do not model the possibility that firms can trade permits in a secondary market. Nevertheless, the allocation of permits is efficient because of the assumption of symmetry, and allowing for trade will not change our results. The unit cost of production is $c$, which we assume to be constant in the base model. In Section 7.3 , we extend our results to the case where the cost of production is impacted by abatement efforts. We consider two scenarios of imperfect competition in the end-product market. In the first case, each firm is a local monopoly and faces an independent, inverse demand function $p_{i}=a-b y_{i}$. In the second case, the $n$ firms compete in a Cournot fashion and face an inverse total demand function $p_{i}=p=\hat{a}-\hat{b} Y$, where $Y=\sum_{i=1}^{n} y_{i}$. In practice, unused permits can be banked for future use or trade. We therefore assume a terminal value of $u$ per unused permit. This value could represent either the value of an unused permit in a secondary market or the net present value of benefits accruing from future use of the permit. We assume that the penalty for not having the requisite number of permits to account for emissions is large enough so that noncompliance is deterred. For example, only 4 of the more than 2000 units affected by the US Acid Rain Program were short of permits to cover their emissions for the 2004 compliance year (EPA 2005).

The optimization problem of firm $i=1, \ldots, n$, is

$$
\begin{gathered}
\operatorname{Maximize}_{\left\{\mu_{i}, \beta_{i}(e), y_{i}\right\}} \Pi_{i}=p_{i} y_{i}+\left[\beta_{i}-\frac{\alpha}{\left(1+\mu_{i}\right)} y_{i}\right]^{+} u \\
\quad-\xi \mu_{i}^{2}-e \beta_{i}-c y_{i} \\
\text { Subject to: } \frac{\alpha}{\left(1+\mu_{i}\right)} y_{i} \leq \beta_{i}, y_{i}, \mu_{i} \geq 0 .
\end{gathered}
$$

The constraint $\left[\alpha /\left(1+\mu_{i}\right)\right] y_{i} \leq \beta_{i}$ implies that each firm must have sufficient permits to account for emissions. Denote $\tilde{\alpha}_{i}:=\alpha /\left(1+\mu_{i}\right)$. We drop the subscript $i$ where unambiguous, for notational and typographic convenience. We focus on symmetric subgame perfect Nash equilibria.

\section{Independent Demands}

We begin with the independent demands case in which firms compete for scarce permits but do not compete in the end-product market. Analysis of each of the subgames follows. 


\subsection{Production Subgame}

Given abatement levels and a permit allocation vector, a representative firm's optimization problem in the third stage of the game is

$$
\begin{array}{r}
\operatorname{Maximize}_{\{y\}} \Pi=(a-b y) y+[\beta-\tilde{\alpha} y]^{+} u \\
-\xi \mu^{2}-e \beta-c y
\end{array}
$$$$
\text { Subject to: } \tilde{\alpha} y \leq \beta, y \geq 0 \text {. }
$$

$\Pi$ is concave in $y ; y^{*}:=\min \{(a-c-\tilde{\alpha} u) / 2 b, \beta / \tilde{\alpha}\}$ is the profit-maximizing quantity, assuming $u<(a$ $-c) / \tilde{\alpha}$. Since each unit produced results in $\tilde{\alpha}$ units of emissions, $\beta / \tilde{\alpha}$ is the maximum production quantity that keeps emissions within the allowable limit. ( $c$ $+\tilde{\alpha} u)$ can be interpreted as the "effective" marginal cost of production, which is the sum of the variable $\operatorname{cost} c$ and the opportunity cost $\tilde{\alpha} u$ since a terminal value equal to $\tilde{\alpha} u$ is lost as a result of producing one unit of output and emitting $\tilde{\alpha}$ units of pollutant. An upper bound on the incremental contribution from a permit is $(a-c) / \tilde{\alpha}$. If $u$ exceeds this bound then there is no incentive to engage in production in the third stage and firms would trivially salvage permits.

Note that the emissions constraint binds when $\beta / \tilde{\alpha}$ $<(a-c-\tilde{\alpha} u) / 2 b$, i.e., when $\beta<\bar{\beta}:=\tilde{\alpha}(a-c$ $-\tilde{\alpha} u) / 2 b$. Firm profit can then be written as follows:

Case (i). When $\beta<\bar{\beta}, y^{*}=\beta / \tilde{\alpha}$, and equilibrium firm profit is

$$
\Pi_{C}^{*}=\left[\frac{(a-c)}{\tilde{\alpha}}-e\right] \beta-\frac{b}{\tilde{\alpha}^{2}} \beta^{2}-\xi \mu^{2} .
$$

Case (ii). When $\beta \geq \bar{\beta}, y^{*}=(a-c-\tilde{\alpha} u) / 2 b$, and equilibrium firm profit is

$$
\Pi_{U}^{*}=\psi+\beta(u-e),
$$

where $\psi=(1 / b)[a-(c+\tilde{\alpha} u) / 2]^{2}-\xi \mu^{2}$ is the unconstrained profit from production, given the abatement level chosen in the first stage.

\subsection{Auction Subgame}

Firms bid for permits in a uniform price sealed-bid auction organized by the regulator. In order to determine its bidding strategy, a firm must anticipate the outcome of the production subgame where one permit is redeemed for each unit of emissions generated in the process of production. Thus, the value placed by a firm on a permit in the auction is determined endogenously by the marginal revenue associated with that permit in the subsequent production subgame. This is in contrast to most of the auctions literature where the value placed by a bidder on the item(s) being auctioned is specified exogenously or is assumed to be drawn from an exogenous distribution. We elaborate on the construction of the "marginal value function" and discuss its properties.
4.2.1. Marginal Value Function. Let $v(\beta)$ denote the firm's marginal revenue from an additional permit when it owns $\beta$ permits, i.e., $\partial \Pi_{C}^{*} / \partial \beta=v(\beta)-e$. From (1) and (2), we have

when $\beta<\bar{\beta}$,

$$
v(\beta)=\frac{(a-c)}{\tilde{\alpha}}-\frac{2 b}{\tilde{\alpha}^{2}} \beta:=\sigma-\lambda \beta,
$$

and when $\beta \geq \bar{\beta}$,

$$
v(\beta)=u,
$$

where $\sigma=(a-c) / \tilde{\alpha}$ and $\lambda=2 b / \tilde{\alpha}^{2}$ are constants, given the abatement level chosen in the first stage. Note that $\sigma-\lambda \bar{\beta}=u$ and when $\beta<\bar{\beta}, v(\beta)>u$. The case when $\beta \geq \bar{\beta}$ is trivial because the emissions constraint does not bind and firms' production decisions are unaffected by the availability of permits. The value then placed on a permit is simply its terminal value. We therefore assume for the remainder of the paper that firms operate in the region defined by $\beta_{i}$ $<\bar{\beta}_{i} \forall i$ and that their beliefs are also restricted to this region. This is a key assumption and essentially implies that each firm faces a capacity constraint because of scarce emissions permits in the production subgame. Under any effective cap and trade program, the availability of permits would constrain profit-maximization or "business as usual" and would result in a market price for permits.

Thus, the value function is restricted to be $v(\beta)=\sigma$ $-\lambda \beta$. Since the emissions constraint binds, the marginal value of a permit is the shadow price corresponding to the emissions constraint. Note that (a $-c) / \tilde{\alpha}$ is the marginal value of a permit when the abatement level is $\mu$ and no permits are held. Given the abatement level, as the number of permits secured $(\beta)$ increases, we get closer to the unconstrained profitmaximizing quantity. The marginal value of a permit thus decreases at the rate $2 b / \tilde{\alpha}^{2}$. The function $v(\beta)$ is crucial in establishing an equilibrium of the auction and in understanding the trade-offs inherent in firm strategies for compliance. We therefore elaborate on its behavior below.

Properties of $v(\beta)$.

i. $\partial[v(\beta)] / \partial \mu \gtrless 0$ for $\mu \lessgtr[\alpha(a-c)-4 b \beta] / 4 b \beta$. Since firms are capacity constrained by the availability of permits, the attractiveness of expanding capacity through permits increases in initial abatement levels. However, for $\mu>[\alpha(a-c)$ $-4 b \beta] / 4 b \beta$, capacities are relatively large and capacity increases through permits yield decreasing value.

ii. $\partial[v(\beta)] / \partial \alpha \gtrless 0$ for $\alpha \lessgtr[4 b \beta(1+\mu)] /(a-c)$. As the capacity constraint gets tighter with increasing $\alpha$, a firm would be willing to pay more for an 
additional permit. Beyond a threshold, however, emissions per unit of output are relatively high and additional permits do not significantly expand capacity, leading to declining value of permits (see Figure 1). Also, $\partial[v(\beta)] / \partial \beta=-[2 b(1$ $\left.+\mu)^{2}\right] / \alpha^{2}<0$ and $\left.\partial^{2}[v(\beta)] / \partial \beta \partial \alpha\right\}=[4 b(1$ $\left.+\mu)^{2}\right] / \alpha^{3}>0$, implying that as $\alpha$ increases the permit allocation $\beta$ has a decreasing influence on the valuation of permits.

iii. $v(\beta)$ decreases in the unit cost of production $c$; i.e., $\partial[v(\beta)] / \partial c=-(1 / \tilde{\alpha})<0$. For higher cost of production, contribution per increment of output is lower and hence a lower value is placed on permits.

iv. $v(\beta)$ decreases in the price sensitivity of demand $b$; i.e., $\partial[v(\beta)] / \partial b=-\left(2 \beta / \tilde{\alpha}^{2}\right)<0$. For higher price sensitivity, the larger drop in price from an additional unit of production results in a lower marginal value of permits.

4.2.2. Equilibrium Bidding Strategy. We are now ready to establish an equilibrium of the second-stage game in which firms participate in a sealed-bid uniform price share auction for emissions permits. Each firm derives its marginal value function as discussed above. A strategy of a firm specifies a schedule of prices for varying fractions of the total available permits $B$. The regulator sets the price such that demand equals supply. As in Wilson (1979), we assume that no firm has proprietary information about demand, emissions, and cost functions and, therefore, about the derived marginal value functions. Symmetry implies that firms have a common marginal value function for permits. Lemma 1 summarizes the equilibrium results.

Lemma 1. For the share auction in the second stage, it is an optimal strategy for a firm to submit a schedule such that at each price e, the requested number of permits is $\beta(e)=B[1$ $-2 e /(n \sigma-\lambda B)] /(n-1)$. The equilibrium price is $e^{*}=$

\section{Figure 1 Marginal Value of a Permit, $v(\beta)$, versus $\alpha$} $(a=2000, b=5, c=200, \mu=0.5)$.

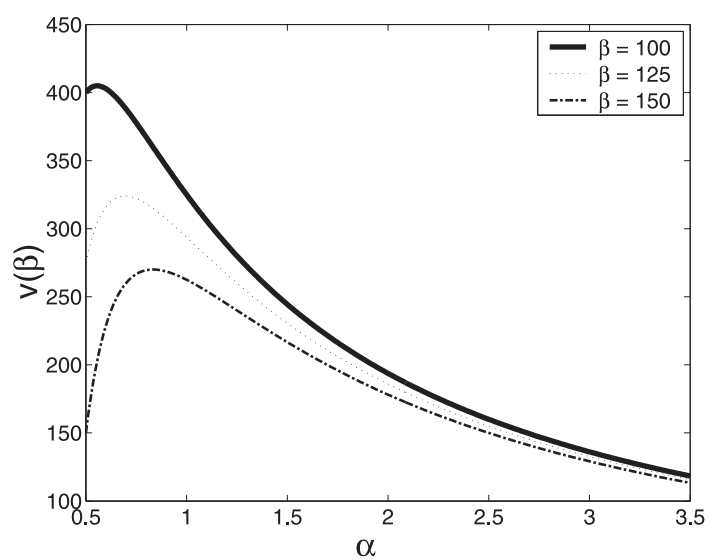

$\frac{1}{2}[\sigma-\lambda(B / n)]$, and the equilibrium number of permits received by each firm is $\beta^{*}=B / n$.

Proof. The proposed equilibrium satisfies $n \cdot \beta\left(e^{*}\right)$ $=B$. We show that if the $(n-1)$ firms other than firm $i$ submit the schedule $\beta(e)$, then it is optimal for firm $i$ to also submit the same schedule $\beta(e)$. Assume that firms $j \neq i$ submit the schedule $\beta(e)$ and $i$ submits some schedule $\tau(e)$. The clearing price $e^{*}$ satisfies

$$
\tau\left(e^{*}\right)=B-(n-1) \beta\left(e^{*}\right)
$$

and firm $i^{\prime}$ s profit is

$$
\begin{gathered}
{\left[\int_{0}^{\tau\left(e^{*}\right)}(\sigma-\lambda z) d z\right]-e^{*} \cdot \tau\left(e^{*}\right)=\left.\left(\sigma z-\lambda \frac{z^{2}}{2}\right)\right|_{0} ^{\left[2 e^{*} B /(n \sigma-\lambda B)\right]}} \\
-e^{*} \cdot\left[\frac{2 e^{*} B}{n \sigma-\lambda B}\right]=\sigma\left[\frac{2 e^{*} B}{n \sigma-\lambda B}\right] \\
-2 \lambda\left[\frac{e^{*} B}{n \sigma-\lambda B}\right]^{2}-\left[\frac{2 e^{* 2} B}{n \sigma-\lambda B}\right] .
\end{gathered}
$$

The function on the right-hand side of (5) is concave in $e^{*}$ and is maximized with respect to $e^{*}$ when

$$
2 \sigma B(n \sigma-\lambda B)-4 \lambda B^{2} e^{*}-4 B(n \sigma-\lambda B) e^{*}=0,
$$

i.e., when $e^{*}=\frac{1}{2}[\sigma-\lambda(B / n)]$. This is exactly the price that will result if firm $i$ submits the schedule $\beta(e)$. The result follows.

We have thus derived a valuable piece of information from this subgame, namely, equilibrium permit price. A methodological contribution of our paper lies in the generalization of Wilson's (1979) analysis of share auctions. In Wilson (1979), the value of a share is proportional to the share fraction. But, as is the case with auctions for emissions permits, it is more likely that the marginal value a bidder places on the items being auctioned decreases with the share fraction. Although we do not establish uniqueness of our equilibrium solution, it is the only plausible one that can be deduced [also see Wilson (1979)]. If asymmetric equilibria do exist in this symmetric game, they are not likely to be intuitively reasonable.

\subsection{Abatement Subgame}

In the first stage of the game, each firm decides the level of abatement, anticipating the equilibrium number of permits it will secure in the auction and the (constrained) output it will be able to produce. Substituting the equilibrium permit price and permit share from Lemma 1 into (1), a firm's optimization problem can be written as

$$
\operatorname{Maximize}_{\{\mu\}} \Pi_{C}^{*}=\frac{B(a-c)(1+\mu)}{2 \alpha n}-\xi \mu^{2}
$$


Subject to: $\mu \geq 0$.

Proposition 1 provides the equilibrium abatement level.

Proposition 1. $\Pi_{C}^{*}$ is concave in $\mu . \mu^{*}=B(a-c) /$ $4 \xi \alpha n$ uniquely maximizes $\Pi_{C}^{*}$.

Proof. See Appendix A.

Table 4 in Appendix B summarizes equilibrium results. Proposition 2 describes the effects of two important parameters in our model-the number of available permits $B$ and the state of current technology manifested in the emissions $\alpha$ per production unit-on equilibrium abatement $\mu^{*}$, permit price $e^{*}$, output $y^{*}$, and profit $\Pi_{C}^{*}$.

Proposition 2. Assume that the emissions constraint binds for all firms. We have

a. $\partial y^{*} / \partial B>0 ; \partial \Pi_{C}^{*} / \partial B>0 ; \partial \mu^{*} / \partial B>0$;

b. $\partial y^{*} / \partial \alpha<0 ; \partial \Pi_{C}^{*} / \partial \alpha<0 ; \partial \mu^{*} / \partial \alpha<0 ; \exists \alpha_{e_{1}}$ such that $\partial e^{*} / \partial \alpha<0$ for $\alpha>\alpha_{e_{1}}$;

c. $\partial^{2} y^{*} / \partial B \partial \alpha<0 ; \partial^{2} \Pi_{C}^{*} / \partial B \partial \alpha<0 ; \partial^{2} \mu^{*} / \partial B \partial \alpha<0$.

Proof. See Appendix A.

The behavior of equilibrium output and profit with respect to the total number of permits $B$ is intuitive. As $B$ increases, each firm secures a larger number of permits in the auction, leading to higher effective capacity, higher output, and profit. However, the behavior of equilibrium abatement with respect to the number of available permits is not so straightforward. It might be expected that abatement and permits to pollute are substitute strategies for compliance; i.e., when more permits become available, firms would engage in lower levels of abatement (Buonanno et al. 2001). However, since firms are assumed to be always constrained by permits, an increase in the number of available permits expands capacity, and the larger capacity makes investment in abatement more attractive since emissions reductions apply to a larger number of production units.

Ceteris paribus, firms in a dirtier industry produce lower output and make smaller profits than firms in a cleaner industry, and altering the number of available permits influences output and profit to a lesser extent in a dirtier industry than in a cleaner industry. Moreover, firms in dirtier industries invest less in abatement (in absolute terms) than firms in cleaner industries. This behavior may appear surprising, but can be understood when we recall that the emissions constraint effectively acts as a capacity constraint on firms' output decisions. Dirty firms (with large $\alpha$ ) face a relatively tighter constraint than cleaner firms and thus produce lower output, making a lower level of investment optimal. We also note that as the regulator varies the total number of permits (thus varying the stringency of the policy), cleaner firms adjust their abatement investments to a larger extent than dirty firms. This is related to the valuation of permits (see Figure 1). Capacity expansion through incremental permits or abatement does not bring as much benefit at higher levels of $\alpha$.

\section{Cournot Competition}

Strategic interactions in oligopoly markets can significantly affect investments in capacity and R\&D (Montero 2002). In this section, we analyze situations where firms not only compete for permits in the auction but also compete for customer demand in a Cournot fashion.

\subsection{Production Subgame}

The optimization problem faced by a representative firm in the third stage of the game is

$$
\begin{array}{r}
\operatorname{Maximize}_{\{y\}} \Pi=(\hat{a}-\hat{b} Y) y+[\beta-\tilde{\alpha} y]^{+} u \\
-\xi \mu^{2}-e \beta-c y,
\end{array}
$$

$$
\text { Subject to: } \tilde{\alpha} y \leq \beta, y \geq 0
$$

where $Y=\sum_{i=1}^{n} y_{i}$. The unconstrained equilibrium output is $\tilde{y}=(\hat{a}-c-\tilde{\alpha} u) / \hat{b}(n+1)$, assuming $u<(\hat{a}$ $-c) / \tilde{\alpha}$. We again focus on situations where the emissions constraint binds for all firms, i.e., when $\beta / \tilde{\alpha}<(\hat{a}$ $-c-\tilde{\alpha} u) / \hat{b}(n+1)$ \{or equivalently $\beta<\breve{\beta}$, where $\breve{\beta}$ $=\tilde{\alpha}[(\hat{a}-c-\tilde{\alpha} u) / \hat{b}(n+1)]\}$, in which case Proposition 3 applies.

Proposition 3. Assume that the emissions constraint binds for all firms. If the abatement level chosen by each firm in the investment stage is symmetrically $\mu$, and the number of permits secured by each firm in the auction stage is symmetrically $\beta$, the equilibrium output of each firm in the third stage of the game where firms compete in a Cournot fashion is $y^{*}=\beta / \tilde{\alpha}$.

Proof. The term $\beta / \tilde{\alpha}$ corresponds to the capacity constraint on a representative firm's production. In the absence of this constraint, the equilibrium output would be $\tilde{y}$. Since firm profit increases with output in the range $[0, \tilde{y}]$, each firm produces up to capacity in equilibrium; the resulting equilibrium output in the third stage is $y^{*}=\beta / \tilde{\alpha}$.

Let $\hat{\Pi}_{C}^{*}$ denote the equilibrium profit when the emissions constraint binds and $\hat{\Pi}_{U}^{*}$ denote the unconstrained equilibrium profit. Let $Z$ denote the total output of all other firms. We then have:

Case (i). When $\beta<\breve{\beta}, y^{*}=\beta / \tilde{\alpha}$, and

$$
\hat{\Pi}_{C}^{*}=-\frac{\hat{b}}{\tilde{\alpha}^{2}} \beta^{2}+\left[\frac{(\hat{a}-c-\tilde{\alpha} e)-\hat{b} Z}{\tilde{\alpha}}\right] \beta-\xi \mu^{2} .
$$


Case (ii). When $\beta \geq \breve{\beta}, y^{*}=(\hat{a}-c-\tilde{\alpha} u) / \hat{b}(n+1)$, and

$$
\begin{aligned}
\hat{\Pi}_{U}^{*}=\left\{\hat{a}-\hat{b} n\left[\frac{\hat{a}-c-\tilde{\alpha} u}{\hat{b}(n+1)}\right]\right\}\left[\frac{\hat{a}-c-\tilde{\alpha} u}{\hat{b}(n+1)}\right] \\
+\left\{\beta-\tilde{\alpha}\left[\frac{\hat{a}-c-\tilde{\alpha} u}{\hat{b}(n+1)}\right]\right\} u-\xi \mu^{2}-e \beta \\
-c\left[\frac{\hat{a}-c-\tilde{\alpha} u}{\hat{b}(n+1)}\right]=\hat{\psi}+\beta(u-e),
\end{aligned}
$$

where $\hat{\psi}=(1 / \hat{b})[(\hat{a}-c+\tilde{\alpha} u) /(n+1)]^{2}-\xi \mu^{2}$ is the unconstrained profit from production, given the abatement level chosen in the first stage.

\subsection{Auction Subgame}

As in Section 4.2, a firm's bidding strategy in the permit auction is deduced from its marginal value function $v(\beta)$. We have:

When $\beta<\breve{\beta}$,

$$
v(\beta)=\left[\frac{(\hat{a}-c)-\hat{b} Z}{\tilde{\alpha}}\right]-\frac{2 \hat{b}}{\tilde{\alpha}^{2}} \beta .
$$

Substituting $Z=(n-1)(\beta / \tilde{\alpha})$ from Proposition 3, we have

$$
v(\beta)=\frac{(\hat{a}-c)}{\tilde{\alpha}}-\frac{\hat{b}(n+1)}{\tilde{\alpha}^{2}} \beta:=\hat{\sigma}-\hat{\lambda} \beta,
$$

when $\beta \geq \breve{\beta}$,

$$
v(\beta)=u,
$$

where $\hat{\sigma}=(\hat{a}-c) / \tilde{\alpha}$ and $\hat{\lambda}=\hat{b}(n+1) / \tilde{\alpha}^{2}$ are constants, given the abatement levels chosen in the first stage. $\hat{\sigma}-\hat{\lambda} \breve{\beta}=u$ and when $\beta<\breve{\beta}, v(\beta)>u$. The behavior of the value function in the case of Cournot competition is similar to that in the case of independent demands. In addition, the marginal value of a permit decreases in the number of competing firms. As the market becomes more competitive, the endproduct price approaches the marginal cost of production; incremental units of output are therefore less valuable. We are now in a position to deduce a firm's optimal bidding strategy in the auction.

LEMma 2. For the share auction in the second stage, it is an optimal strategy for a firm to submit a schedule such that at each price $e$, the requested number of permits is $\beta(e)$ $=B[1-2 e /(n \hat{\sigma}-\hat{\lambda} B)] /(n-1)$. The equilibrium price is $\hat{e}^{*}=\frac{1}{2}[\hat{\sigma}-\hat{\lambda}(B / n)]$, and the equilibrium number of permits received by each firm is $\hat{\beta}^{*}=B / n$.

Proof. See Appendix A.

Substituting the equilibrium permit price and permit share from Lemma 2 into the profit function in (6), we have

$$
\hat{\Pi}_{C}^{*}=\frac{B \alpha n(\hat{a}-c)(1+\mu)-\hat{b} B^{2}(n-1)(1+\mu)^{2}}{2 \alpha^{2} n^{2}}-\xi \mu^{2} .
$$

\subsection{Abatement Subgame}

In the abatement subgame, a firm maximizes the profit function in (10) with respect to abatement $\mu$. Proposition 4 provides the equilibrium abatement level.

Proposition 4. $\hat{\Pi}_{C}^{*}$ in (10) is concave in $\mu . \hat{\mu}^{*}=$ $\left\{\left[B \alpha n(\hat{a}-c)-2 \hat{b} B^{2}(n-1)\right] /\left[2 \hat{b} B^{2}(n-1)+4 \xi \alpha^{2} n^{2}\right]\right\}$ uniquely maximizes $\hat{\Pi}_{C}^{*}$.

Proof. See Appendix A.

To ensure a nonnegative equilibrium value of abatement, we write $\hat{\mu}^{*}=\max \left\{\left[B \alpha n(\hat{a}-c)-2 \hat{b} B^{2}(n\right.\right.$

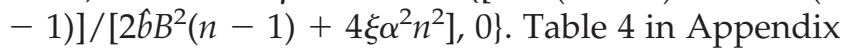
$B$ summarizes equilibrium results. Proposition 5 formally describes the behavior of equilibrium abatement $\hat{\mu}^{*}$, permit price $\hat{e}^{*}$, output $\hat{y}^{*}$, and profit $\hat{\Pi}_{C}^{*}$ with respect to $B$ and $\alpha$.

Proposition 5. Assume that the emissions constraint binds for all firms.

For $\alpha<2 \hat{b} B(n-1) / n(\hat{a}-c)$, we have $\hat{\mu}^{*}=0$ and

a. $\partial \hat{y}^{*} / \partial B>0 ; \partial \hat{\Pi}_{C}^{*} / \partial B<0$;

b. $\partial \hat{y}^{*} / \partial \alpha<0 ; \partial \hat{\Pi}_{C}^{*} / \partial \alpha>0 ; \exists \alpha_{\hat{e}_{1}}$ such that $\partial \hat{e}^{*} / \partial \alpha \gtrless$ 0 for $\alpha \lessgtr \alpha_{\hat{e}_{1}}$;

c. $\partial^{2} \hat{y}^{*} / \partial B \partial \alpha<0 ; \partial^{2} \hat{\Pi}_{C}^{*} / \partial B \partial \alpha>0$.

For $\alpha>2 \hat{b} B(n-1) / n(\hat{a}-c)$, we have $\hat{\mu}^{*}=[B \alpha n(\hat{a}-c)$ $\left.-2 \hat{b} B^{2}(n-1)\right] /\left[2 \hat{b} B^{2}(n-1)+4 \xi \alpha^{2} n^{2}\right]$ and

d. $\partial \hat{y}^{*} / \partial B>0 ; \partial \hat{\Pi}_{C}^{*} / \partial B>0 ; \exists \alpha_{\hat{\mu}_{1}}$ such that $\partial \hat{\mu}^{*} / \partial B$ $\lessgtr 0$ for $\alpha \lessgtr \alpha_{\hat{\kappa}_{1}} ;$

e. $\partial \hat{y}^{*} / \partial \alpha<0 ; \partial \prod_{C}^{*} / \partial \alpha<0 ; \exists \alpha_{\hat{\mu}_{1}}$ such that $\partial \hat{\mu}^{*} / \partial \alpha$ $\gtrless 0$ for $\alpha \lessgtr \alpha_{\hat{\mu}_{1}} ; \exists \alpha_{\hat{e}_{2}}$ such that $\partial \hat{e}^{*} / \partial \alpha<0$ for $\alpha$ $>\alpha_{\hat{e}_{2}} ;$

f. $\exists \alpha_{\hat{y}_{1}}$ such that $\partial^{2} \hat{y}^{*} / \partial B \partial \alpha<0$ for $\alpha>\alpha_{\hat{\vartheta}_{1}} ; \exists \alpha_{\hat{\Pi}_{C}}$ such that $\partial^{2} \hat{\Pi}_{C}^{*} / \partial B \partial \alpha<0$ for $\alpha>\alpha_{\hat{\Pi}_{C_{1}}} ; \alpha_{\hat{\mu}_{2}}$ such that $\left.\partial^{2} \hat{\mu}^{*} / \partial B \partial \alpha\right)<0$ for $\alpha>\alpha_{\hat{\mu}_{2}}$.

Proof. See Appendix A.

The behavior of output with respect to $B$ and $\alpha$ is intuitive and the explanation is similar to that in Section 4. However, the behavior of abatement and firm profit with respect to $B$ and $\alpha$ is not so obvious. Note that a firm would invest in abatement only if the marginal benefit it obtains from that investment exceeds the marginal cost. From (10) we have

$$
\frac{\partial \hat{\Pi}_{C}^{*}}{\partial \mu}=\frac{(\hat{a}-c)}{2}\left(\frac{B}{\alpha n}\right)-\hat{b}(n-1)(1+\mu)\left(\frac{B}{\alpha n}\right)^{2}-2 \xi \mu .
$$

That is, the maximum marginal benefit a firm can obtain from incremental abatement is $[(\hat{a}-c) / 2](B /$ $\alpha n)$, at $\mu=0$, where $B / \alpha n$ is the equilibrium output level and corresponds to the capacity constraint faced 
by the firm. This maximum marginal benefit is the same as that in the independent demands case (see proof of Proposition 1). However, when firms are Cournot competitors, expanded production capacity from abatement has a negative externality on firm profit; as capacity expands, the price of output drops and has an adverse impact on profit. A marginal increase in abatement thus leads to a decline in marginal benefit, proportional to $\hat{b}(n-1)(B / \alpha n)^{2}$. When $[(\hat{a}$ $-c) / 2](B / \alpha n)<\hat{b}(n-1)(B / \alpha n)^{2}$ or, equivalently, when $\alpha<2 \hat{b} B(n-1) / n(\hat{a}-c)$, the firm's net marginal benefit from an increase in abatement is negative. Thus, relatively clean firms invest zero in abatement. In fact, if the nonnegativity constraint on abatement is relaxed, firms would have an incentive to increase their emissions and thus limit production capacity when $\alpha$ $<2 \hat{b} B(n-1) / n(\hat{a}-c)$.

Figure 2 depicts the behavior of equilibrium firm profit (at the equilibrium levels of output, permit price, and abatement) with respect to $\alpha$ for different values of $B$. Recall that our analysis applies when the emissions constraint is binding for all firms. For very low values of $\alpha$ (not depicted in Figure 2), the emissions constraint does not bind and firm profit is invariant with respect to $\alpha$. For relatively clean firms [i.e., $\alpha<2 \hat{b} B(n-1) / n(\hat{a}-c)$ ] that have a binding emissions constraint, a decrease in the number of available permits intriguingly results in an increase in equilibrium profit. As the emissions constraint gets tighter, firms restrict their output. Lower industry output drives product prices up and, in a Cournot environment, results in an increase in firm profit. A similar explanation applies to the increase in firm profit in this region as $\alpha$ increases.

However, beyond a certain threshold [i.e., $\alpha>2 \hat{b} B(n$ $-1) / n(\hat{a}-c)]$, firms' emissions per unit of output are large relative to the emissions cap; the capacity constraint is therefore stiffer and firms benefit from in-

Figure 2 Firm Profit, $\hat{\Pi}_{\sigma}^{*}$, versus $\alpha$ $(\hat{a}=50000, \hat{b}=125, c=200, \xi=50000, n=25)$.

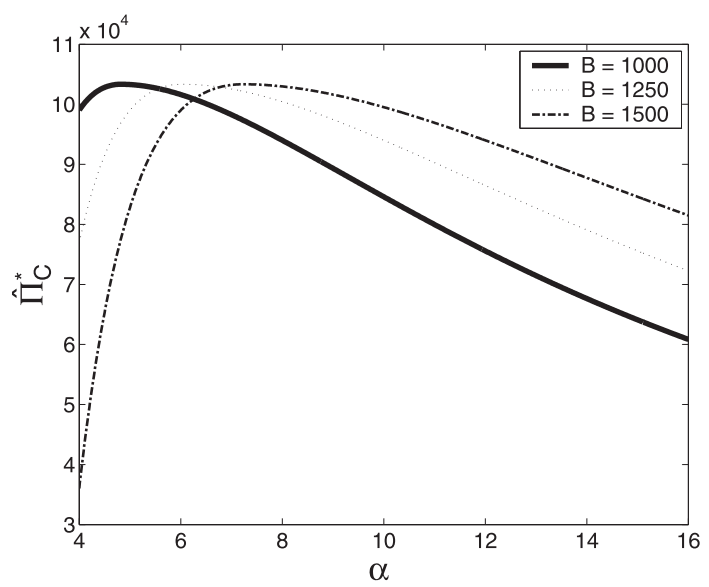

creases in $B$. Positive investments in abatement result and firm profits increase in $B$. Beyond a point, abatement tapers off with respect to $\alpha$ and is influenced less by changes in $B$; the explanation for this behavior is similar to that detailed for the independent demands case in Section 4.

\section{Numerical Example}

Table 1 includes a numerical example that highlights the results obtained in Sections 4 and 5. Note here that the total number of permits $B$ constrains profit-maximization both when firms are clean $(\alpha=4)$ and when firms are dirty $(\alpha=10)$. In the case of independent demands, as $B$ increases, each firm secures more permits, and equilibrium output and profit increase. Equilibrium abatement increases with the supply of permits, which is driven by the assumption that firms are constrained in profit-maximization by the availability of permits. For the same supply of permits, we observe higher output and profit when firms are clean $(\alpha=4)$ than when firms are dirty $(\alpha=10)$. It can be verified that equilibrium output and profit are elastic with respect to $\alpha$, that is, $\left(\partial y^{*} / \partial \alpha\right) \cdot\left(\alpha / y^{*}\right)<-1$ and $\left(\partial \Pi_{C}^{*} / \partial \alpha\right) \cdot\left(\alpha / \Pi_{C}^{*}\right)<-1$. This implies that output and profit can increase significantly as firms get cleaner. Indeed, when $B=150,000$, we find a $281 \%$ increase in output and a $230 \%$ increase in profit when $\alpha$ decreases by $60 \%$ from 10 to 4 . Abatement levels are higher when firms are cleaner. As seen in Table 1, for any level of permit supply, the abatement levels for $\alpha=4$ are greater than those for $\alpha=10$. It can also be verified that firm profit is elastic to the supply of permits, i.e., $\left(\partial \Pi_{C}^{*} / \partial B\right) \cdot\left(B / \Pi_{C}^{*}\right)>1$. A $33 \%$ decrease in $B$ from 150,000 to 100,000 results in a $42 \%$ decrease in profit when $\alpha=4$ and a 38\% decrease in profit when $\alpha=10$. A related result is that output is elastic to the supply of permits, i.e., $\left(\partial y^{*} / \partial B\right) \cdot\left(B / y^{*}\right)>1$. This is a useful result from a regulatory standpoint because an increasing stringency has significant impact on firm output and, hence, on the level of emissions.

In the case of Cournot competition, when $\alpha=4$, an increase in the supply of permits results in a decrease in firm profit. This is due to the increase in industry capacity. The unit price of output drops as a result, as does firm profit, and firms respond by not engaging in abatement. In contrast to the independent demands case, we observe that abatement drops to zero when the supply of permits goes up from 100,000 to 150,000 for $\alpha=4$. Under Cournot competition, increasing stringency can be beneficial to firms when they are relatively clean. From Table 1 , we observe that when $B$ is reduced from 200,000 to 150,000 , firm profit increases by $10 \%$ when $\alpha=4$ but decreases by $13 \%$ when $\alpha=10$. Production levels are higher and abatement levels are lower when $\alpha=4$ than when $\alpha=10$. However, when 


\begin{tabular}{|c|c|c|c|c|c|c|c|c|c|c|}
\hline \multirow[b]{4}{*}{$B$} & \multicolumn{5}{|c|}{ Independent demands } & \multicolumn{5}{|c|}{ Competition } \\
\hline & \multicolumn{5}{|c|}{$(n=250, a=2000, b=0.3, c=200, \xi=50000)$} & \multicolumn{5}{|c|}{$(n=250, \hat{a}=2000, \hat{b}=0.03, c=200, \xi=50000)$} \\
\hline & \multicolumn{5}{|c|}{$\alpha=4$} & \multicolumn{5}{|c|}{$\alpha=4$} \\
\hline & $\mu^{*}$ & $y^{\star}$ & $e^{\star}$ & $\beta^{*}$ & $\Pi_{C}^{*}$ & $\hat{\mu}^{*}$ & $\hat{y}^{*}$ & $\hat{e}^{\star}$ & $\hat{\beta}^{*}$ & $\Pi_{C}^{*}$ \\
\hline 100,000 & 0.9000 & 190.00 & 400.43 & 400 & $130,500.00$ & 0.0876 & 108.76 & 133.37 & 400 & $53,319.98$ \\
\hline 150,000 & 1.3500 & 352.50 & 466.62 & 600 & $226,125.00$ & 0 & 150.00 & 83.81 & 600 & $53,002.89$ \\
\hline 200,000 & \multicolumn{5}{|c|}{$\alpha=10$} & \multicolumn{5}{|c|}{$\alpha=10$} \\
\hline$B$ & $\mu^{*}$ & $y^{\star}$ & $e^{\star}$ & $\beta^{*}$ & $\Pi_{C}^{*}$ & $\hat{\mu}^{*}$ & $\hat{y}^{*}$ & $\hat{e}^{\star}$ & $\hat{\beta}^{*}$ & $\Pi_{C}^{*}$ \\
\hline 100,000 & 0.3600 & 54.40 & 120.18 & 400 & $42,480.00$ & 0.2148 & 48.59 & 87.11 & 400 & $32,606.83$ \\
\hline 150,000 & 0.5400 & 92.40 & 134.33 & 600 & $68,580.00$ & 0.2136 & 72.82 & 75.95 & 600 & $43,449.55$ \\
\hline
\end{tabular}

the supply of permits is large, it is possible that firm profit can be greater in a dirty industry than in a cleaner one, due to the industry capacity argument made above. When $B=200,000$, firm profit is $48,294.88$ for $\alpha=4$ and 50,075.77 for $\alpha=10$. For relatively large $\alpha$, it can be shown that the elasticities for the case of Cournot competition are structurally similar to those described above for the case of independent demands.

\section{Extensions}

In order to test the sensitivity of our results to assumptions in the model, we explore four extensions-an emissions function in which the abatement level is independent of the volume of production, grandfathering of permits in addition to auctioning, abatement efforts impacting the cost of production, and firm heterogeneity. Our model is quite robust to these variations, with our structural results largely continuing to hold. Proofs are omitted for brevity.

\subsection{Emissions Function}

We consider an emissions function of the form $\alpha y-S$, where the abatement level $S$ is chosen in the first stage of the game at a cost $\xi S^{2}$. Such an emissions function represents investments made toward fixed-capacity abatement, as opposed to investments that result in per-unit reduction in emissions considered in the base model. Examples include efforts to capture and sequester exhaust gases in fixed capacity geologic formations (Brennan and Burruss 2003) — an avenue that is being actively considered for sequestering $\mathrm{CO}_{2}$. Equilibrium results are presented in Table 5 in Appendix B. Proposition 6 summarizes the main results for the case of independent demands.

Proposition 6.

a. $\partial y^{*} / \partial B>0 ; \partial \Pi_{C}^{*} / \partial B>0 ; \partial S^{*} / \partial B<0$.

b. $\partial y^{*} / \partial \alpha<0 ; \partial \Pi_{C}^{*} / \partial \alpha<0 ; \exists \alpha_{S_{1}}$ such that $\partial S^{*} / \partial \alpha$ $\gtrless 0$ for $\alpha \lessgtr \alpha_{S_{1}} ; \exists \alpha_{e_{2}}$ such that $\partial e^{*} / \partial \alpha<0$ for $\alpha>\alpha_{e_{2}}$. c. $\partial^{2} y^{*} / \partial B \partial \alpha<0 ; \partial^{2} S^{*} / \partial B \partial \alpha>0 ; \exists \alpha_{\Pi_{C_{1}}}$ such that $\partial^{2} \Pi_{C}^{*} / \partial B \partial \alpha<0$ for $\alpha>\alpha_{\Pi_{C_{1}}}$.

A comparison with Proposition 2 shows that all structural attributes of our results are preserved except for the behavior of equilibrium abatement. Recall that, in the base model, abatement impacted emissions per unit of output; the effective production capacity for a firm was $B(1+\mu) / \alpha$ (i.e., multiplicative in $\mu$ ). Therefore, abatement and permits were complementary strategies and abatement increased with permit availability. Here, however, abatement reduces emissions by a fixed amount, independent of the output level; the effective capacity is given by $(B+S) / \alpha$ (i.e., additive in $S$ ). Therefore, abatement and permits are substitute strategies and greater permit availability decreases abatement. Thus, the effect of stringency on abatement investment by firms depends upon the type of abatement firms have recourse to. Proposition 7 summarizes the results for the case of Cournot competition.

\section{Proposition 7.}

a. $\partial \hat{y}^{*} / \partial B>0 ; \partial \hat{S}^{*} / \partial B<0 ; \exists \alpha_{\hat{\Pi}_{C_{2}}}, \alpha_{\Lambda_{C_{3}}}$ such that $\partial \hat{\Pi}_{C}^{*} / \partial B<0$ for $\alpha<\alpha_{\hat{\Pi}_{C_{2}}}, n>3$, and $\partial \hat{\Pi}_{C}^{*} / \partial B$ $>0$ for $\alpha>\alpha_{\hat{\Pi}_{C_{C}}}$.

b. $\partial \hat{y}^{*} / \partial \alpha<0 ; \exists \alpha_{\widehat{S}_{1}}$ such that $\partial \hat{S}^{*} / \partial \alpha \gtrless 0$ for $\alpha \lessgtr \alpha_{\widehat{S}_{1}}$; $\exists \alpha_{\hat{\Pi}_{C_{4}}}$ such that $\partial \hat{\Pi}_{C}^{*} / \partial \alpha<0$ for $\alpha>\alpha_{\hat{\Pi}_{C_{4}}} ; \exists \alpha_{\hat{e}_{3}}$ such that $\partial \hat{e}^{*} / \partial \alpha<0$ for $\alpha>\alpha_{\hat{e}_{3}}$.

c. $\partial^{2} \hat{S}^{*} / \partial B \partial \alpha>0 ; \exists \alpha_{\hat{y}_{2}}$ such that $\partial^{2} \hat{y}^{*} / \partial B \partial \alpha<0$ for $\alpha>\alpha_{\hat{y}_{2}} ; \exists \alpha_{\hat{\Pi}_{C_{5}}}$ such that $\partial^{2} \hat{\Pi}_{C}^{*} / \partial B \partial \alpha<0$ for $\alpha>\alpha_{\hat{\Pi}_{C_{5}}}$.

In particular, we note that with competition in the end-product market, firms in a relatively clean industry benefit from a reduction in the number of available permits. As discussed earlier, an increase in the number of permits increases industry capacity and exerts downward pressure on the price of output and, hence, on the profitability of clean firms. 


\subsection{Grandfathering in Addition to Auctioning}

As discussed earlier, the regulator could possibly grandfather permits based on historical emissions or process inputs. Grandfathering can be incorporated into our model by allowing a fraction $\gamma$ of the total number of permits $B$ to be grandfathered per firm, with the remaining fraction $1-\gamma$ offered via the auction. Working again by backward induction with the assumption that scarcity of permits constrains profit maximization, the equilibrium output has a similar functional form (given abatement levels) as before. Although the marginal value of permits decreases with grandfathering, the smaller number of permits now available in the auction offsets the decrease in the value of permits, and the equilibrium permit price too has a similar functional form (given abatement levels) as before. However, as expected, equilibrium abatement is lower due to the availability of grandfathered, free permits. Equilibrium results (see Table 6 in Appendix B) change accordingly, but most of our structural results are preserved. Proposition 8 summarizes results for the case of independent demands.

\section{Proposition 8.}

a. $\partial y^{*} / \partial B>0 ; \partial \Pi_{C}^{*} / \partial B>0 ; \exists \alpha_{\mu_{1}}^{G}$ such that $\partial \mu^{*} / \partial B$ $\lessgtr 0$ for $\alpha \lessgtr \alpha_{\mu_{1}}^{G}$.

b. $\partial y^{*} / \partial \alpha<0 ; \partial \prod_{C}^{*} / \partial \alpha<0 ; \exists \alpha_{\mu_{1}}^{G}$ such that $\partial \mu^{*} / \partial \alpha$ $\gtrless 0$ for $\alpha \lessgtr \alpha_{\mu_{1}}^{G} ; \exists \alpha_{e_{1}}^{G}$ such that $\partial e^{*} / \partial \alpha<0$ for $\alpha$ $>\alpha_{e_{1}}^{G}$.

A comparison of Propositions 8 and 2 shows that the behavior of equilibrium output, permit price, and profit remains unchanged with the introduction of grandfathering. However, the behavior of equilibrium abatement changes. Abatement always increases with permit availability in the independent demands case of the base model. However, the availability of free, grandfathered permits increases effective production capacity significantly when $\alpha$ is low and makes further capacity expansion through abatement less valuable. For larger $\alpha$, the capacity constraint is stiffer and abatement increases with permit availability-as in the base model. The behavior of abatement with respect to $\alpha$ is similar. Proposition 9 summarizes results for the case of Cournot competition.

\section{Proposition 9.}

For $\alpha<[2 \hat{b} B(n-1)+2 \hat{b} B \gamma(n+1)] /[n(\hat{a}-c)(1+\gamma)]$, we have $\hat{\mu}^{*}=0$ and

$$
\begin{aligned}
& \text { a. } \partial \hat{y}^{*} / \partial B>0 ; \partial \hat{\Pi}_{C}^{*} / \partial B<0 ; \\
& \text { b. } \partial \hat{y}^{*} / \partial \alpha<0 ; \partial \hat{\Pi}_{C}^{*} / \partial \alpha>0 ; \exists \alpha_{\hat{e}_{1}}^{G} \text { such that } \partial \hat{e}^{*} / \partial \alpha \\
& \quad \gtrless 0 \text { for } \alpha \lessgtr \alpha_{\hat{e}_{1}}^{G} .
\end{aligned}
$$

For $\alpha>[2 \hat{b} B(n-1)+2 \hat{b} B \gamma(n+1)] /[n(\hat{a}-c)(1+\gamma)]$, we have $\hat{\mu}^{*}=\left[\operatorname{B\alpha n}(\hat{a}-c)(1+\gamma)-2 \hat{b} B^{2}(n-1)\right.$

$\left.-2 \hat{b} B^{2} \gamma(n+1)\right] /\left[2 \hat{b} B^{2}(n-1)+2 \hat{b} B^{2} \gamma(n+1)\right.$

$\left.+4 \xi \alpha^{2} n^{2}\right]$ and c. $\partial \hat{y}^{*} / \partial B>0 ; \exists \alpha_{\hat{\Pi}_{c}{ }^{G}} ;$ such that $\partial \hat{\Pi}_{C}^{*} / \partial B \lessgtr 0$ for $\alpha$

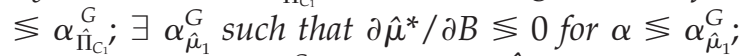

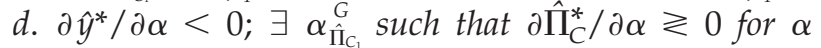
$\lessgtr \alpha_{\hat{\Pi}_{c_{1}}^{G}}^{G} ; \exists \alpha_{\hat{\mu}_{1}}^{G}$ such that $\partial \hat{\mu}^{*} / \partial \alpha \gtrless 0$ for $\alpha \lessgtr \alpha_{\hat{\mu}_{1}}^{G} ; \exists$ $\alpha_{\hat{e}_{2}}^{G}$ such that $\partial \hat{e}^{*} / \partial \alpha<0$ for $\alpha>\alpha_{\hat{e}_{2}}^{G}$.

A comparison of Propositions 9 (parts c and d) and 5 (parts $\mathrm{d}$ and e) indicates that the availability of free permits accentuates the distinctive effects of $B$ and $\alpha$ on the profits of firms competing in a Cournot fashion.

\subsection{Abatement and the Cost of Production}

Abatement efforts might involve the implementation of resource-efficient processing of materials, translating into a decrease in unit production cost. On the other hand, stipulations on emissions levels could necessitate more sophisticated processing of materials or the use of more expensive, cleaner materials to mitigate emissions, leading to an increase in unit production cost. We can incorporate such effects into the base model by replacing the constant unit cost of production $c$ with the function $c+\rho \mu$ where $\rho \in(-c / \mu, \infty)$ is a constant and $\mu$ is the abatement level. Equilibrium results are presented in Table 7 in Appendix B. Our structural results remain unchanged.

\subsection{Firm Heterogeneity}

In this section, we relax the assumption of symmetry. Since the game becomes necessarily complicated, we restrict our analysis to a two-firm game and explore the effects of heterogeneity in dirtiness levels $\alpha_{i}$, costs of abatement $\xi_{i}$, and costs of production $c_{i}, i=1,2$.

7.4.1. Independent Demands. We maintain the assumption that firms are constrained in profit maximization by the availability of permits. The equilibrium output of firm $i$ in the third stage is $y_{i}^{*}:=\beta_{i}(1$ $\left.+\mu_{i}\right) / \alpha_{i}$. Denote $\sigma_{i}=\left(a-c_{i}\right)\left(1+\mu_{i}\right) / \alpha_{i}$ and $\lambda_{i}=2 b(1$ $\left.+\mu_{i}\right)^{2} / \alpha_{i}^{2}$. The marginal value function for permits is $v\left(\beta_{i}\right)=\sigma_{i}-\lambda_{i} \beta_{i}$. In the auction stage, firm $i$ 's equilibrium bidding strategy is $\beta_{i}(e)=B-\left[2\left(\sigma_{j}-\sigma_{i}\right.\right.$ $\left.\left.+\lambda_{i} B\right) e\right] /\left[\sigma_{i} \lambda_{j}+\sigma_{j} \lambda_{i}-\lambda_{i} \lambda_{j} B\right], j \neq i$. Equilibrium permit price is $e^{*}=\left[\sigma_{i} \lambda_{j}+\sigma_{j} \lambda_{i}-\lambda_{i} \lambda_{j} B\right] /\left[2\left(\lambda_{i}+\lambda_{j}\right)\right]$ and the equilibrium number of permits received by firm $i$ is $\beta_{i}^{*}:=\beta_{i}\left(e^{*}\right)=\left[\sigma_{i}-\sigma_{j}+\lambda_{j} B\right] /\left(\lambda_{i}+\lambda_{j}\right)$. Note that $\beta_{1}^{*}$ $+\beta_{2}^{*}=B$. The profit function $\Pi_{i}$, after incorporating equilibrium output, permit price, and permit share, is a function of both $\mu_{i}$ and $\mu_{j}$. Equilibrium values of abatement correspond to the intersection of the two reaction functions $\mu_{1}^{*}\left(\mu_{2}\right)$ and $\mu_{2}^{*}\left(\mu_{1}\right)$. Analytical derivation and treatment of these reaction functions is intractable and we therefore present a numerical example in Table 2.

7.4.2. Cournot Competition. Equilibrium output of firm $i$ is $\hat{y}_{i}^{*}:=\left[\beta_{i}\left(1+\mu_{i}\right)\right] / \alpha_{i}$. Denote $\hat{\sigma}_{i}=\left[\left(\hat{a}-c_{i}\right)(1\right.$ $\left.\left.+\mu_{i}\right)\right] / \alpha_{i}, \hat{\lambda}_{i}=\left[2 \hat{b}\left(1+\mu_{i}\right)^{2} \alpha_{j}-\hat{b}\left(1+\mu_{i}\right)\left(1+\mu_{j}\right) \alpha_{i}\right] /$ $\alpha_{i}^{2} \alpha_{j}$, and $\omega=\left[\hat{b} B\left(1+\mu_{i}\right)\left(1+\mu_{j}\right)\right] / \alpha_{i} \alpha_{j}$. The marginal 
Table 2

Two-Firm Asymmetric Game: Numerical Example (Independent Demands)

\begin{tabular}{|c|c|c|c|c|c|c|c|c|c|}
\hline Scenario & $\mu_{1}^{*}$ & $\mu_{2}^{*}$ & $y_{1}^{*}$ & $y_{2}^{*}$ & $e^{\star}$ & $\beta_{1}^{*}$ & $\beta_{2}^{*}$ & $\Pi_{1}^{*}$ & $\Pi_{2}^{*}$ \\
\hline \multicolumn{10}{|l|}{ Asymmetry in dirtiness $^{\mathrm{a}}$} \\
\hline$\alpha_{1}=4, \alpha_{2}=6$ & 0.5263 & 0.4233 & 91.66 & 37.90 & 168.54 & 240.22 & 159.78 & $68,644.99$ & $25,153.45$ \\
\hline$\alpha_{1}=4, \alpha_{2}=8$ & 0.4716 & 0.2811 & 108.90 & 16.66 & 130.79 & 296.00 & 104.00 & $86,887.47$ & $11,037.92$ \\
\hline \multicolumn{10}{|l|}{ Asymmetry in abatement costs ${ }^{b}$} \\
\hline$\xi_{1}=30000, \xi_{2}=50000$ & 0.7896 & 0.4929 & 66.55 & 44.00 & 169.18 & 223.14 & 176.86 & $41,195.39$ & $27,458.88$ \\
\hline$\xi_{1}=30000, \xi_{2}=70000$ & 0.7945 & 0.3572 & 71.43 & 36.46 & 162.35 & 238.84 & 161.16 & $45,352.56$ & $23,876.44$ \\
\hline \multicolumn{10}{|l|}{ Asymmetry in production costs ${ }^{\mathrm{c}}$} \\
\hline$c_{1}=100, c_{2}=200$ & 0.5794 & 0.5222 & 59.27 & 44.36 & 172.06 & 225.16 & 174.84 & $39,521.26$ & $26,287.56$ \\
\hline$c_{1}=100, c_{2}=300$ & 0.5829 & 0.4707 & 66.09 & 36.64 & 163.45 & 250.52 & 149.48 & $45,957.49$ & $20,065.64$ \\
\hline \multicolumn{10}{|l|}{ Asymmetry in dirtiness and production costs ${ }^{\mathrm{d}}$} \\
\hline$\alpha_{1}=4, \alpha_{2}=6 ; c_{1}=300, c_{2}=100$ & 0.5000 & 0.4904 & 76.45 & 48.73 & 175.46 & 203.81 & 196.19 & $52,461.53$ & $34,271.74$ \\
\hline$\alpha_{1}=5, \alpha_{2}=6 ; c_{1}=275, c_{2}=100$ & 0.5172 & 0.5436 & 57.40 & 54.24 & 174.63 & 189.16 & 210.84 & $36,131.29$ & $36,755.20$ \\
\hline \multicolumn{10}{|l|}{ Asymmetry in dirtiness, varying stringency ${ }^{\mathrm{e}}$} \\
\hline$B=400 ; \alpha_{1}=4, \alpha_{2}=8$ & 0.4716 & 0.2811 & 108.90 & 16.66 & 130.79 & 296.00 & 104.00 & $86,887.47$ & $11,037.92$ \\
\hline$B=350 ; \alpha_{1}=4, \alpha_{2}=8$ & 0.4734 & 0.2416 & 107.89 & 8.86 & 132.80 & 292.91 & 57.09 & $85,899.31$ & 5056.17 \\
\hline
\end{tabular}

value function for permits is $v\left(\beta_{i}\right)=\left(\hat{\sigma}_{i}-\omega\right)-\hat{\lambda}_{i} \beta_{i}$. An equilibrium bidding strategy is $\beta_{i}(e)=B-\left[2\left(\hat{\sigma}_{j}-\hat{\sigma}_{i}\right.\right.$ $\left.\left.+\hat{\lambda}_{i} B\right) e\right] /\left[\hat{\sigma}_{i} \hat{\lambda}_{j}+\hat{\sigma}_{j} \hat{\lambda}_{i}-\hat{\lambda}_{i} \hat{\lambda}_{j} B-\omega\left(\hat{\lambda}_{i}+\hat{\lambda}_{j}\right)\right]$. Equilibrium permit price is $\hat{e}^{*}=\left[\hat{\sigma}_{i} \hat{\lambda}_{j}+\hat{\sigma}_{j} \hat{\lambda}_{i}-\hat{\lambda}_{i} \hat{\lambda}_{j} B-\omega\left(\hat{\lambda}_{i}+\hat{\lambda}_{j}\right)\right] /$ $\left[2\left(\hat{\lambda}_{i}+\hat{\lambda}_{j}\right)\right]$ and the equilibrium number of permits received by firm $i$ is $\hat{\beta}_{i}^{*}:=\beta_{i}\left(\hat{e}^{*}\right)=\left(\hat{\sigma}_{i}-\hat{\sigma}_{j}+\hat{\lambda}_{j} B\right) /$ $\left(\hat{\lambda}_{i}+\hat{\lambda}_{j}\right)$. The profit function $\hat{\Pi}_{i}$, after incorporating equilibrium output, permit price, and permit share, is a function of both $\mu_{i}$ and $\mu_{j}$. Equilibrium values of abatement correspond to the intersection of the reaction functions $\hat{\mu}_{1}^{*}\left(\mu_{2}\right)$ and $\hat{\mu}_{2}^{*}\left(\mu_{1}\right)$.

Interestingly, we observe behavior analogous to that described in Sections 4 and 5. In both demand scenarios, we observe that as a firm gets dirtier, it abates less, produces less, and obtains a smaller profit. As expected, a firm abates less when its cost of abate- ment or cost of production goes up. In addition, there is evidence of a trade-off among firm cleanliness, the corresponding cost of production, and firm profit. In the example presented in Table 2, the cleaner firm has a higher cost of production. In the combination $\alpha_{1}=4$, $c_{1}=300, \alpha_{2}=6, c_{2}=100$, the cleaner firm, i.e., firm 1 , secures a larger profit, thus demonstrating that it can pay to be clean even if the unit cost of production is higher. But in the combination $\alpha_{1}=5, c_{1}=275, \alpha_{2}=6$, $c_{2}=100$, the dirtier firm, i.e., firm 2, secures a larger profit. A similar trade-off is observed in Table 3. In the case of independent demands, firm profits increase in the number of available permits. However, the profit of the dirtier firm is more sensitive, in percentage terms, to changes in the number of permits. In Table 2, when $B$ is reduced from 400 to 350 , the cleaner firm's

Table 3 Two-Firm Asymmetric Game: Numerical Example (Cournot Competition)

\begin{tabular}{|c|c|c|c|c|c|c|c|c|c|}
\hline Scenario & $\hat{\mu}_{1}^{*}$ & $\hat{\mu}_{2}^{*}$ & $\hat{y}_{1}^{*}$ & $\hat{y}_{2}^{*}$ & $\hat{e}^{\star}$ & $\hat{\beta}_{1}^{*}$ & $\hat{\beta}_{2}^{*}$ & $\hat{\Pi}_{1}^{*}$ & $\hat{\Pi}_{2}^{*}$ \\
\hline \multicolumn{10}{|l|}{ Asymmetry in dirtiness ${ }^{a}$} \\
\hline$\alpha_{1}=2, \alpha_{2}=4$ & 0.1895 & 0.4325 & 113.42 & 74.96 & 86.55 & 190.69 & 209.31 & $79,025.29$ & $36,855.05$ \\
\hline$\alpha_{1}=2, \alpha_{2}=6$ & 0.1134 & 0.2579 & 133.12 & 33.73 & 83.56 & 239.12 & 160.88 & $107,937.24$ & $15,805.79$ \\
\hline \multicolumn{10}{|l|}{ Asymmetry in abatement costs ${ }^{b}$} \\
\hline$\xi_{1}=30000, \xi_{2}=50000$ & 0.6940 & 0.4969 & 87.36 & 72.50 & 119.42 & 206.26 & 193.74 & $48,335.77$ & $37,071.91$ \\
\hline$\xi_{1}=30000, \xi_{2}=70000$ & 0.6960 & 0.3839 & 90.43 & 64.60 & 121.41 & 213.28 & 186.72 & $52,249.57$ & $33,218.40$ \\
\hline \multicolumn{10}{|l|}{ Asymmetry in production costs ${ }^{c}$} \\
\hline$c_{1}=100, c_{2}=200$ & 0.5830 & 0.5006 & 91.08 & 63.72 & 132.69 & 230.16 & 169.84 & $55,026.77$ & $30,307.15$ \\
\hline$c_{1}=100, c_{2}=300$ & 0.5798 & 0.4209 & 103.18 & 49.28 & 122.78 & 261.27 & 138.73 & $68,505.84$ & $20,318.78$ \\
\hline \multicolumn{10}{|l|}{ Asymmetry in dirtiness and production costs ${ }^{\mathrm{d}}$} \\
\hline$\alpha_{1}=2, \alpha_{2}=6 ; c_{1}=300, c_{2}=100$ & 0.1684 & 0.3731 & 112.46 & 47.49 & 98.73 & 192.49 & 207.51 & $80,820.29$ & $24,802.71$ \\
\hline$\alpha_{1}=5, \alpha_{2}=6 ; c_{1}=275, c_{2}=100$ & 0.5685 & 0.6855 & 51.62 & 66.14 & 137.72 & 164.57 & 235.43 & $19,833.87$ & $30,797.88$ \\
\hline \multicolumn{10}{|l|}{ Asymmetry in dirtiness, varying stringency } \\
\hline$B=400 ; \alpha_{1}=2, \alpha_{2}=6$ & 0.1134 & 0.2579 & 133.12 & 33.73 & 83.56 & 239.12 & 160.88 & $107,937.24$ & $15,805.79$ \\
\hline$B=350 ; \alpha_{1}=2, \alpha_{2}=6$ & 0.1264 & 0.1920 & 137.71 & 20.96 & 89.58 & 244.51 & 105.49 & $115,924.82$ & 9804.01 \\
\hline
\end{tabular}


profit goes down by just $1 \%$, whereas the dirtier firm's profit goes down by $54 \%$. In the case of competition, the cleaner firm's profit increases when the number of permits is reduced. In Table 3 , when $B$ is reduced from 400 to 350 , the cleaner firm's profit goes up by $7 \%$, whereas the dirtier firm's profit goes down by $38 \%$. Thus, in either demand scenario, the impact of increasing stringency on profit is far less severe on the cleaner firm than on the dirtier firm.

\section{Discussion and Future Work}

The crux of any market-based program for pollution control is to allow firms to flexibly apply compliance levers. This paper presents a methodology by which a firm can derive its marginal value function for permits and translate this value function into an optimal bidding strategy in the auction for permits. In addition, the modeling effort provides a means for firms to deduce their optimal levels of abatement and output. The model used in this paper is a fairly simple representation of investment in abatement, permit bidding and allocation, and production. Yet the interplay among the elements in the model illustrates that the behavior of equilibrium outcomes is intricate. The findings in this paper can aid regulators in understanding how abatement levels, permit prices, and industry output interact. At first glance, it might seem that changing the total number of permits would influence abatement to a greater extent in a dirtier industry than in a cleaner one. However, we find the opposite to be true. Our model also suggests that equilibrium permit price can be low in a relatively dirty industry because of a stiffer capacity limit, resulting in a decrease in the value placed on permits, a lower permit price in the auction, and lower production levels. Reductions in the number of available permits drive firms in a relatively dirty industry to lower output levels. Industry structure influences equilibrium outcomes. Interestingly, in the case of Cournot competition in the end-product market, a reduction in the number of permits offered in the auction can increase equilibrium firm profit in a relatively clean industry. This is favorable from the viewpoint of a regulator aiming to increase policy stringency. Traditionally, firms lobby to roll back pollution standards because of the lingering belief that stricter environmental regulations erode competitiveness (Porter and van der Linde 1995). But, in a competitive setting, an excessive number of permits can be detrimental to firm profit because of resultingly large industry capacity that brings down product price and firm profit.

A promising observation is that our results are reasonably robust to modeling assumptions. That structural behavior is preserved in our asymmetric twoplayer game is especially encouraging. However, several extensions to our work merit consideration in future research. The timing of decisions in our model represents situations where abatement investment decisions are long-term in nature and occur at a slower rate than the allocation of permits. However, in cases where the abatement investment cycle is relatively faster (e.g., as could be the case with switching from high-sulfur to low-sulfur coal) or where the potential cost of abatement investments drive decisions to lobby against emissions caps, a sequence of decisions where the allocation of permits precedes the abatement decision might be more appropriate. Firms' incentives to lobby against emissions limits, given abatement costs and forecasts of future environmental regulation, can be modeled as in Lyon and Maxwell (2004). Our analysis treated imperfect, quantity competition. It would be interesting to see how the results change with perfect competition in the output market or when firms decide on prices rather than quantities. Uncertainty can be incorporated in end-product demand and, hence, in the valuation of permits by firms. Uncertainty can also be introduced in the regulator's choice of the emissions cap, in beliefs about the cost structures of competitors, or in the likelihood of abatement resulting from investment efforts. In practice, permits can be banked for future use and investments in abatement yield emissions reductions across multiple time periods; an extension to multiple periods will therefore be very insightful. Emissions permits are also exchanged in markets outside of auctions through brokered trades, electronic screens, or direct trades between participants without an intermediary. Interactions between permit auctions and such exchange mechanisms are worth exploring. According to our model, dirty firms find it cheaper at the margin to reduce output rather than invest more in abatement in response to increasing stringency. Regulators might not necessarily desire output reductions or plant closures-outcomes that involve a variety of social costs-but might prefer that firms make investments in cleaning up their technologies and thus privately bear the costs of reducing emissions. At the heart of this discussion is the question of how many permits the regulator should issue. Although we do not address this important question, our construct may be of use as a "what if" tool for the social planner to assess the impact that the emissions cap has on firm profitability, output, and abatement investment compared to the social costs and benefits of allowing a certain amount of pollution. Finally, in the spirit of Bovenberg, Goulder, and Gurney (2004) and Smith, Ross, and Montgomery (2002), our model could be extended to incorporate the regulator's choice of the fraction of permits to grandfather in order to preserve "equity values" in the affected industry. 


\section{Acknowledgments}

We thank the editors and anonymous referees for their helpful comments. We also thank Dr. William Lovejoy (Ross School of Business, University of Michigan), Dr. Richard Loulou (GERAD, McGill University), William Rogers (Air Programs, Detroit Edison Co.), Constantine Blathras (US EPA Region 5), and participants in INFORMS meetings, CU Environmental and Resource Economics workshops, and seminars at the Ross School of Business for their feedback. We acknowledge the financial support of The Erb Institute and the Natural Sciences and Engineering Research Council of Canada.

\section{Appendix A}

\section{Proofs}

Proof of Proposition 1.

$$
\begin{gathered}
\frac{d \Pi_{C}^{*}}{d \mu}=\frac{B(a-c)}{2 \alpha n}-2 \xi \mu \\
\frac{d^{2} \Pi_{C}^{*}}{d \mu^{2}}=-2 \xi
\end{gathered}
$$

Implying that $\Pi_{C}^{*}$ is concave in $\mu . d \Pi_{C}^{*} / d \mu=0 \Rightarrow \mu^{*}=B(a$ - c) $/ 4 \xi \alpha n$, the profit-maximizing abatement level.

Proof of Proposition $2 a$.

i. $\partial y^{*} / \partial B=[4 \xi \alpha n+2 B(a-c)] / 4 \xi \alpha^{2} n^{2}>0$.

ii. $\partial \Pi_{C}^{*} / \partial B=[(a-c) / 2 \alpha n]+\left[B(a-c)^{2} / 8 \xi \alpha^{2} n^{2}\right]>0$.

iii. $\partial \mu^{*} / \partial B=(a-c) / 4 \xi \alpha n>0$, since $a>c+\tilde{\alpha} u$.

Proof of Proposition $2 b$.

i. $\partial y^{*} / \partial \alpha=-\left(B / \alpha^{2} n\right)-B^{2}(a-c) / 2 \xi \alpha^{3} n^{2}<0$.

ii. $\partial \Pi_{C}^{*} / \partial \alpha=-\left[B(a-c) / 2 \alpha^{2} n\right]-\left[B^{2}(a-c)^{2} / 8 \xi \alpha^{3} n^{2}\right]$ $<0$.

iii. $\partial \mu^{*} / \partial \alpha=-\left[B(a-c) / 4 \xi \alpha^{2} n\right]<0$.

iv. $\partial e^{*} / \partial \alpha=\left\{\left[-2 \xi^{2} \alpha^{3} n^{3}(a-c)+8 b B \xi^{2} \alpha^{2} n^{2}+6 b B^{2} \xi \alpha n(a\right.\right.$ $\left.\left.-c)+b B^{3}(a-c)^{2}-B \xi \alpha^{2} n^{2}(a-c)^{2}\right] / 4 \xi^{2} \alpha^{5} n^{3}\right\} ;$ $-2 \xi^{2} \alpha^{3} n^{3}(a-c)+8 b B \xi^{2} \alpha^{2} n^{2}+6 b B^{2} \xi \alpha n(a-c)<0$ for $\alpha>B\left[4 b \xi+2 \sqrt{4 b^{2} \xi^{2}+3 b \xi(a-c)^{2}}\right] / 2 \xi n(a-c)$, and $b B^{3}(a-c)^{2}-B \xi \alpha^{2} n^{2}(a-c)^{2}<0$ for $\alpha>$ $\sqrt{b B^{2} / \xi n^{2}}$. Hence, $\partial e^{*} / \partial \alpha<0$ for $\alpha>\max \{B[4 b \xi$ $\left.\left.+2 \sqrt{4 b^{2} \xi^{2}+3 b \xi(a-c)^{2}}\right] / 2 \xi n(a-c), \sqrt{b B^{2} / \xi n^{2}}\right\}$.

Proof of Proposition 2c.

i. $\partial^{2} y^{*} / \partial B \partial \alpha=-\left(1 / \alpha^{2} n\right)-[B(a-c)] / \xi \alpha^{3} n^{2}<0$

ii. $\partial^{2} \Pi_{C}^{*} / \partial B \partial \alpha=-\left[(a-c) / 2 \alpha^{2} n\right]-\left[B(a-c)^{2} / 8 \xi \alpha^{3} n^{2}\right]$ $<0$.

iii. $\partial^{2} \mu^{*} / \partial B \partial \alpha=-\left[(a-c) / 4 \xi \alpha^{2} n\right]<0$.

Proof of Lemma 2. The proposed equilibrium satisfies $n \cdot \beta\left(\hat{e}^{*}\right)=B$. We show that if the $(n-1)$ firms other than firm $i$ submit the schedule $\beta(e)$ then it is optimal for firm $i$ to also submit the same schedule $\beta(e)$. Assume that firms $j \neq i$ submit the schedule $\beta(e)$ and $i$ submits some schedule $\tau(e)$. The clearing price $\hat{e}^{*}$ satisfies

$$
\tau\left(\hat{e}^{*}\right)=B-(n-1) \beta\left(\hat{e}^{*}\right)
$$

and firm $i^{\prime}$ s profit is

$$
\begin{aligned}
& {\left[\int_{0}^{\tau\left(\hat{e}^{*}\right)}(\hat{\sigma}-\hat{\lambda} z) d z\right]-\hat{e}^{*} \cdot \tau\left(\hat{e}^{*}\right)} \\
& \quad=\left.\left(\hat{\sigma} z-\hat{\lambda} \frac{z^{2}}{2}\right)\right|_{0} ^{\left[2 \hat{e}^{*} B /(n \hat{\sigma}-\hat{\lambda} B\right.}-\hat{e}^{*} \cdot\left[\frac{2 \hat{e}^{*} B}{n \hat{\sigma}-\hat{\lambda} B}\right] \\
& \quad=\hat{\sigma}\left[\frac{2 \hat{e}^{*} B}{n \hat{\sigma}-\hat{\lambda} B}\right]-2 \hat{\lambda}\left[\frac{\hat{e}^{*} B}{n \hat{\sigma}-\hat{\lambda} B}\right]^{2}-\left[\frac{2 \hat{e}^{* 2} B}{n \hat{\sigma}-\hat{\lambda} B}\right]
\end{aligned}
$$

The function on the right-hand side of (12) is concave in $\hat{e}^{*}$ and is maximized with respect to $\hat{e}^{*}$ when

$$
2 \hat{\sigma} B(n \hat{\sigma}-\hat{\lambda} B)-4 \hat{\lambda} B^{2} \hat{e}^{*}-4 B(n \hat{\sigma}-\hat{\lambda} B) \hat{e}^{*}=0,
$$

i.e., when $\hat{e}^{*}=\frac{1}{2}[\hat{\sigma}-\hat{\lambda}(B / n)]$. This is exactly the price that will result if firm $i$ submits the schedule $\beta(e)$. The result follows.

Proof of Proposition 4.

$$
\begin{gathered}
\frac{d \hat{\Pi}_{C}^{*}}{d \mu}=\frac{B \alpha n(\hat{a}-c)-2 \hat{b} B^{2}(n-1)(1+\mu)}{2 \alpha^{2} n^{2}}-2 \xi \mu \\
\frac{d^{2} \hat{\Pi}_{C}^{*}}{d \mu^{2}}=-\frac{\hat{b} B^{2}(n-1)}{\alpha^{2} n^{2}}-2 \xi<0
\end{gathered}
$$

Implying that $\hat{\Pi}_{C}^{*}$ is concave in $\mu . d \hat{\Pi}_{C}^{*} / \mathrm{d} \mu=0$ yields $\hat{\mu}^{*}$ $=\left[\operatorname{B\alpha } n(\hat{a}-c)-2 \hat{b} B^{2}(n-1)\right] /\left[2 \hat{b} B^{2}(n-1)+4 \xi \alpha^{2} n^{2}\right]$, the profit-maximizing abatement level.

Proof of Proposition 5a.

i. $\partial \hat{y}^{*} / \partial B=1 / \alpha n>0$.

ii. $\partial \hat{\Pi}_{C}^{*} / \partial B=[(\hat{a}-c) / 2 \alpha n]-\left[2 \hat{b} B(n-1) / 2 \alpha^{2} n^{2}\right]$ $<0$.

Proof of Proposition $5 b$.

i. $\partial \hat{y}^{*} / \partial \alpha=-\left(B / \alpha^{2} n\right)<0$.

ii. $\partial \Pi_{C}^{*} / \partial \alpha=-\left[B(\hat{a}-c) / 2 \alpha^{2} n\right]+\left[\hat{b} B^{2}(n-1) / \alpha^{3} n^{2}\right]$ $>0$.

iii. $\partial \hat{e}^{*} / \partial \alpha=-\left[(\hat{a}-c) / 2 \alpha^{2}\right]+\left(2 \hat{b} B / \alpha^{3} n\right) \gtrless 0$ for $\alpha$ $\lessgtr[4 \hat{b} B / n(\hat{a}-c)]$.

Proof of Proposition 5c.

i. $\partial^{2} \hat{y}^{*} / \partial B \partial \alpha=-\left(1 / \alpha^{2} n\right)<0$.

ii. $\partial^{2} \hat{\Pi}_{C}^{*} / \partial B \partial \alpha=-\left[(\hat{a}-c) / 2 \alpha^{2} n\right]+\left[2 \hat{b} B(n-1) / \alpha^{3} n^{2}\right]$ $>0$.

Proof of Proposition 5d.

i. $\partial \hat{y}^{*} / \partial B=2 \xi \alpha n\left[B \alpha n(\hat{a}-c)-\hat{b} B^{2}(n-1)+2 \xi \alpha^{2} n^{2}\right] /$ $\left[\hat{b} B^{2}(n-1)+2 \xi \alpha^{2} n^{2}\right]^{2}$. From the condition $\beta<\breve{\beta}$ we have $n>\left[\hat{b} B(1+\mu)^{2}\right] /\left[\alpha(\hat{a}-c)(1+\mu)-\hat{b} B(1+\mu)^{2}\right.$ $-\alpha^{2} u$ ], implying $B \alpha n(\hat{a}-c)>\hat{b} B^{2}(1+\mu)(n+1)$ $>\hat{b} B^{2}(n-1)$, and therefore $\partial \hat{y}^{*} / \partial B>0$.

ii. $\partial \hat{\Pi}_{C}^{*} / \partial B=\xi \alpha n\left[4 \xi \alpha^{2} n^{2}(\hat{a}-c)-8 \hat{b} B \xi \alpha n(n-1)+B \alpha n(\hat{a}\right.$ $\left.-c)^{2}-2 \hat{b} B^{2}(\hat{a}-c)(n-1)\right] / 2\left[\hat{b} B^{2}(n-1)+2 \xi \alpha^{2} n^{2}\right]^{2}$ $>0$.

iii. $\partial \hat{\mu}^{*} / \partial B=\alpha n\left[2 \xi \alpha^{2} n^{2}(\hat{a}-c)-8 \hat{b} B \xi \alpha n(n-1)-\hat{b} B^{2}(\hat{a}\right.$ $-c)(n-1)] / 2\left[\hat{b} B^{2}(n-1)+2 \xi \alpha^{2} n^{2}\right]^{2} \lessgtr 0$ for $\alpha \lessgtr B[4 \hat{b} \xi(n$ $\left.-1)+\sqrt{16 b^{2} \xi^{2}(n-1)^{2}+2 \hat{b} \xi(\hat{a}-c)^{2}(n-1)}\right] /[2 \xi n(\hat{a}$ $-c)]$.

Proof of Proposition 5e.

i. $\partial \hat{y}^{*} / \partial \alpha=-\left\{2 B \xi n\left[B \alpha n(\hat{a}-c)-\hat{b} B^{2}(n-1)+2 \xi \alpha^{2} n^{2}\right] /\right.$ $\left.\left[\widehat{b} B^{2}(n-1)+2 \xi \alpha^{2} n^{2}\right]^{2}\right\}$. From the condition $\beta<\breve{\beta}$ we have $n>\left[\hat{b} B(1+\mu)^{2}\right] /\left[\alpha(\hat{a}-c)(1+\mu)-\hat{b} B(1+\mu)^{2}\right.$ 
- $\alpha^{2} u$ ], implying $B \alpha n(\hat{a}-c)>\hat{b} B^{2}(1+\mu)(n+1)$

$>\hat{b} B^{2}(n-1)$, and therefore $\partial \hat{y}^{*} / \partial \alpha<0$.

ii. $\partial \hat{\Pi}_{C}^{*} / \partial \alpha=-\left\{B \xi n\left[4 \xi \alpha^{2} n^{2}(\hat{a}-c)-8 \hat{b} B \xi \alpha n(n-1)\right.\right.$

$\left.+B \alpha n(\hat{a}-c)^{2}-2 \hat{b} B^{2}(\hat{a}-c)(n-1)\right] / 2\left[\hat{b} B^{2}(n-1)\right.$

$\left.\left.+2 \xi \alpha^{2} n^{2}\right]^{2}\right\}<0$.

iii. $\partial \hat{\mu}^{*} / \partial \alpha=-\left\{B n\left[2 \xi \alpha^{2} n^{2}(\hat{a}-c)-8 \hat{b} B \xi \alpha n(n-1)-\hat{b} B^{2}(\hat{a}\right.\right.$

$\left.-c)(n-1)] / 2\left[\hat{b} B^{2}(n-1)+2 \xi \alpha^{2} n^{2}\right]^{2}\right\} \gtrless 0$ for $\alpha$

$\lessgtr B\left[4 \hat{b} \xi(n-1)+\sqrt{16 \hat{b}^{2} \xi^{2}(n-1)^{2}+2 \hat{b} \xi(\hat{a}-c)^{2}(n-1)}\right] /$

$[2 \xi n(\hat{a}-c)]$.

iv. $\partial \hat{e}^{*} / \partial \alpha=-\left\{2 \xi n^{2}\left[2 \xi^{2} \alpha^{4} n^{4}(\hat{a}-c)-4 \hat{b} B \xi^{2} \alpha^{3} n^{3}(n+1)\right.\right.$

$\left.\left.-3 \hat{b} B^{2} \xi \alpha^{2} n^{2}(\hat{a}-c)(n+1)\right] /\left[\hat{b} B^{2}(n-1)+2 \xi \alpha^{2} n^{2}\right]^{3}\right\}$

- $\left\{2 \xi n^{2}\left[B \xi \alpha^{3} n^{3}(\hat{a}-c)^{2}-\hat{b} B^{3} \alpha n(\hat{a}-c)^{2}+\hat{b}^{2} B^{4}(\hat{a}\right.\right.$

$\left.-c)(n-1)] /\left[\hat{b} B^{2}(n-1)+2 \xi \alpha^{2} n^{2}\right]^{3}\right\} ; 2 \xi^{2} \alpha^{4} n^{4}(\hat{a}-c)$

$-4 \hat{b} B \xi^{2} \alpha^{3} n^{3}(n+1)-3 \hat{b} B^{2} \xi \alpha^{2} n^{2}(\hat{a}-c)(n+1)>0$ for $\alpha$

$>B\left[2 \hat{b} \xi(n+1)+\sqrt{4 \hat{b}^{2} \xi^{2}(n+1)^{2}+6 \hat{b} \xi(\hat{a}-c)^{2}(n+1)^{2}}\right] /$

$[2 \xi n(\hat{a}-c)]$, and $B \xi \alpha^{3} n^{3}(\hat{a}-c)^{2}-\hat{b} B^{3} \alpha n(\hat{a}-c)^{2}>0$ for $\alpha>\sqrt{\hat{b} B^{2} / \xi n^{2}}$. Hence, $\partial \hat{e}^{*} / \partial \alpha<0$ for $\alpha>\max \{B[2 \hat{b} \xi(n$

$\left.+1)+\sqrt{4 \hat{b}^{2} \xi^{2}(n+1)^{2}+6 \hat{b} \xi(\hat{a}-c)^{2}(n+1)^{2}}\right] /[2 \xi n(\hat{a}-$

c), $\sqrt{\hat{b} B^{2} / \xi n^{2}}$.

Proof of Proposition $5 f$.

i. $\partial^{2} \hat{y}^{*} / \partial B \partial \alpha=-\left\{2 \xi n\left[4 \xi^{2} \alpha^{4} n^{4}-12 \hat{b} B^{2} \xi \alpha^{2} n^{2}(n-1)\right.\right.$

$\left.+4 B \xi \alpha^{3} n^{3}(\hat{a}-c)-2 \hat{b} B^{3} \alpha n^{2}(\hat{a}-c)\right] /\left[\hat{b} B^{2}(n-1)\right.$

$\left.\left.+2 \xi \alpha^{2} n^{2}\right]^{3}\right\}-\left\{2 \xi n\left[2 \hat{b} B^{3} \alpha n(\hat{a}-c)+\hat{b}^{2} B^{4}(n-1)^{2}\right] /\left[\hat{b} B^{2}(n\right.\right.$
- 1) $\left.\left.+2 \xi \alpha^{2} n^{2}\right]^{3}\right\} ; 4 \xi^{2} \alpha^{4} n^{4}-12 \hat{b} B^{2} \xi \alpha^{2} n^{2}(n-1)>0$ for $\alpha$ $>\sqrt{\left[3 \hat{b} B^{2}(n-1) / \xi n^{2}\right]}$, and $4 B \xi \alpha^{3} n^{3}(\hat{a}-c)-2 \hat{b} B^{3} \alpha n^{2}(\hat{a}$ $-c)>0$ for $\alpha>\sqrt{b B^{2} / 2 \xi n}$. Hence, $\partial^{2} \hat{y}^{*} / \partial B \partial \alpha<0$ for $\alpha$ $>\sqrt{\left[3 \hat{b} B^{2}(n-1) / \xi n^{2}\right]}$, since $n \geq 2$.

ii. $\partial^{2} \hat{\Pi}_{C}^{*} / \partial B \partial \alpha=-\left\{\xi n\left[4 \xi^{2} \alpha^{4} n^{4}(\hat{a}-c)-16 \hat{b} B \xi^{2} \alpha^{3} n^{3}(n-1)\right.\right.$

$\left.\left.-12 \hat{b} B^{2} \xi \alpha^{2} n^{2}(\hat{a}-c)(n-1)\right] /\left[\hat{b} B^{2}(n-1)+2 \xi \alpha^{2} n^{2}\right]^{3}\right\}$

- $\left\{\xi \xi\left[2 B \xi \alpha^{3} n^{3}(\hat{a}-c)^{2}-\hat{b} B^{3} \alpha n(\hat{a}-c)^{2}(n-1)\right] /\left[\hat{b} B^{2}(n\right.\right.$

$\left.\left.-1)+2 \xi \alpha^{2} n^{2}\right]^{3}\right\}-\left\{\xi n\left[8 \hat{b}^{2} B^{3} \xi \alpha n(n-1)^{2}+\hat{b}^{2} B^{4}(\hat{a}-c)(n\right.\right.$

$\left.\left.-1)^{2}\right] /\left[\hat{b} B^{2}(n-1)+2 \xi \alpha^{2} n^{2}\right]^{3}\right\} ; 2 B \xi \alpha^{3} n^{3}(\hat{a}-c)^{2}-\hat{b} B^{3} \alpha n(\hat{a}$

$-c)^{2}(n-1)>0$ for $\alpha>\sqrt{\left[\hat{b} B^{2}(n-1) / 2 \xi n^{2}\right]}$, and $4 \xi^{2} \alpha^{4} n^{4}(\hat{a}-c)-16 \hat{b} B \xi^{2} \alpha^{3} n^{3}(n-1)$ $-12 \hat{b} B^{2} \xi \alpha^{2} n^{2}(\hat{a}-c)(n-1)>0$ for $\alpha>\{B[2 \hat{b} \xi(n$ $\left.-1)+\sqrt{4 \hat{b}^{2} \xi^{2}(n-1)^{2}+3 \hat{b} \xi(\hat{a}-c)^{2}(n-1)}\right] /$ $[\xi n(\hat{a}-c)\}$. Hence, $\partial^{2} \hat{\pi}_{C}^{*} / \partial B \partial \alpha<0$ for $\alpha$ $>\max \left\{\sqrt{\left[\hat{b} B^{2}(n-1) / 2 \xi n^{2}\right]}, \quad B[2 \hat{b} \xi(n-1)\right.$ $\left.+\sqrt{4 \hat{b}^{2} \xi^{2}(n-1)^{2}+3 \hat{b} \xi(\hat{a}-c)^{2}(n-1)}\right] /[\xi n(\hat{a}-c)\}$.

iii. $\partial^{2} \hat{\mu}^{*} / \partial B \partial \alpha=-\left\{n\left[4 \xi^{2} \alpha^{4} n^{4}(\hat{a}-c)-32 \hat{b} B \xi^{2} \alpha^{3} n^{3}(n-1)\right.\right.$ $\left.\left.-12 \hat{b} B^{2} \xi \alpha^{2} n^{2}(\hat{a}-c)(n-1)\right] /\left[2\left(\hat{b} B^{2}(n-1)+2 \xi \alpha^{2} n^{2}\right)\right]^{3}\right\}$ - $\left\{n\left[16 \hat{b}^{2} B^{3} \xi \alpha n(n-1)^{2}+\hat{b}^{2} B^{4} n(\hat{a}-c)(n-2)\right.\right.$ $\left.\left.+\hat{b}^{2} B^{4}(\hat{a}-c)\right] /\left[2\left(\hat{b} B^{2}(n-1)+2 \xi \alpha^{2} n^{2}\right)\right]^{3}\right\}<0$ for $\left[4 \xi^{2} \alpha^{4} n^{4}(\hat{a}-c)-32 \hat{b} B \xi^{2} \alpha^{3} n^{3}(n-1)-12 \hat{b} B^{2} \xi \alpha^{2} n^{2}(\hat{a}\right.$ $-c)(n-1)]>0$, i.e., for $\alpha>\{B[4 \hat{b} \xi(n-1)$ $\left.\left.+\sqrt{16 b^{2} \xi^{2}(n-1)^{2}+3 \hat{b} \xi(\hat{a}-c)^{2}(n-1)}\right] /[\xi n(\hat{a}-c)]\right\}$.

\section{Appendix B}

\section{Tables}

Table 4 Equilibrium Results-Sections 4 and 5 (Base Model)

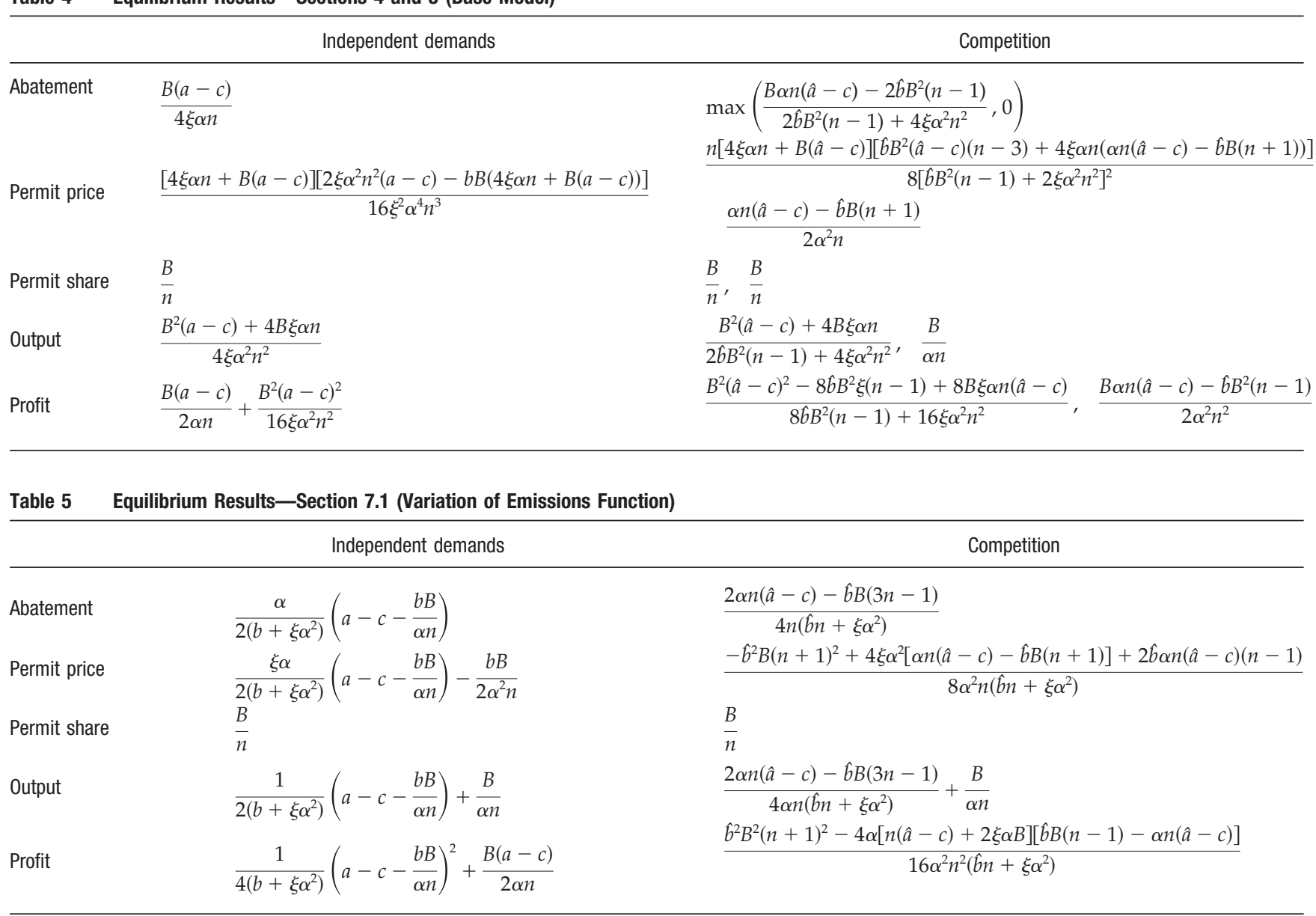

Note. To ensure nonnegative equilibrium values of abatement and output, $(\hat{a}-c)>[\hat{b} B(3 n-1) / 2 \alpha n]$ for the case of competition. 
Table 6

Equilibrium Results-Section 7.2 (Grandfathering)

Independent demands

Competition

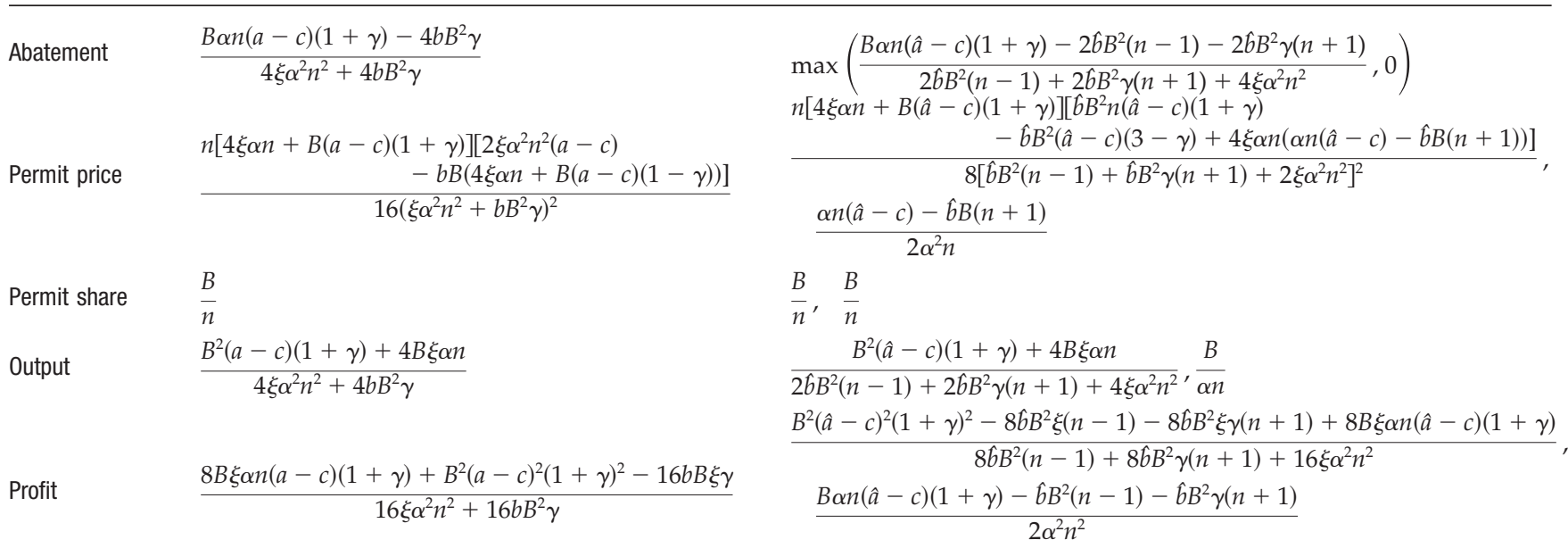

Table 7 Equilibrium Results-Section 7.3 (Abatement and Cost of Production)

\begin{tabular}{|c|c|c|}
\hline & Independent demands & Competition \\
\hline \multirow{2}{*}{ Abatement } & $B(a-c-\rho)$ & $B \alpha n(\hat{a}-c-\rho)-2 \hat{b} B^{2}(n-1)$ \\
\hline & $\overline{2(2 \xi \alpha n+B \rho)}$ & $\overline{2\left(\hat{b} B^{2}(n-1)+2 \xi \alpha^{2} n^{2}+B \alpha n \rho\right)}$ \\
\hline \multirow[t]{2}{*}{ Permit price } & $\begin{array}{l}{[4 \xi \alpha n+B(a-c+\rho)]\left[4 \xi \alpha^{2} n^{2}(a-c)\right.} \\
\quad-8 b B \xi \alpha n-B(a-c+\rho)(2 b B-\alpha n \rho)]\end{array}$ & $\begin{aligned} n[4 \xi \alpha n+B(\hat{a}-c+\rho)][B(\hat{a}-c+\rho)(\alpha n \rho+\hat{b} B n \\
-3 \hat{b} B+4 \xi \alpha n(\alpha n(\hat{a}-c)-\hat{b} B(n+1))]\end{aligned}$ \\
\hline & $8 \alpha^{2} n(2 \xi \alpha n+B \rho)^{2}$ & $8\left[\hat{b} B^{2}(n-1)+2 \xi \alpha^{2} n^{2}+B \alpha n \rho\right]^{2}$ \\
\hline \multirow{2}{*}{ Permit share } & $B$ & $B$ \\
\hline & $\bar{n}$ & $\bar{n}$ \\
\hline \multirow{2}{*}{ Output } & $B^{2}(a-c+\rho)+4 B \xi \alpha n$ & $B^{2}(\hat{a}-c+\rho)+4 B \xi \alpha n$ \\
\hline & $2 \alpha n(2 \xi \alpha n+B \rho)$ & $\overline{2\left[\hat{b} B^{2}(n-1)+2 \xi \alpha^{2} n^{2}+B \alpha n \rho\right]}$ \\
\hline Profit & $\frac{B(a-c)[8 \xi \alpha n+B(a-c+2 \rho)]}{8 \alpha n(2 \xi \alpha n+B \rho)}$ & $\frac{B\left[B(\hat{a}-c)(\hat{a}-c+2 \rho)-8 b B \xi(n-1)+8 \xi \alpha n(\hat{a}-c)+B \rho^{2}\right]}{8\left[\hat{b} B^{2}(n-1)+2 \xi \alpha^{2} n^{2}+B \alpha n \rho\right]^{2}}$ \\
\hline
\end{tabular}

Note. To ensure nonnegative equilibrium values of abatement, $(\hat{a}-c-\rho)>[2 \hat{b} B(n-1) / \alpha n]$ for the case of competition.

\section{References}

Back, K., J. F. Zender. 1993. Auctions of divisible goods: On the rationale for the treasury experiment. The Review of Financial Studies 6(4) 733-764.

Bakal, I. S., E. Akçali. 2006. Effects of random yield in reverse supply chains with price sensitive supply and demand. Production and Operations Management 15(3) 407-420.

Bikhchandani, S., C. Huang. 1993. The economics of treasury securities markets. Journal of Economic Perspectives 7(3) 117-134.

Bovenberg, A. L., L. H. Goulder, D. J. Gurney. 2004. Efficiency costs of meeting industry-distributional constraints under environmental permits and taxes. RAND Journal 36(4) 951-971.

Brennan, S. T., R. C. Burruss. 2003. Specific Sequestration Volumes: A Useful Tool for $\mathrm{CO}_{2}$ Storage Capacity Assessment. U.S. Geological Survey.

Buonanno, P., C. Carraro, E. Castelnuovo, M. Galeotti. 2001. Emission trading restrictions with endogenous technological change. International Environmental Agreements: Politics, Law and Economics 1(3) 379-395.

Burtraw, D. 2000. Innovation under the Tradable Sulfur Dioxide Emissions Permits Program in the U.S. Electricity Sector. Discussion Paper, Resources for the Future, Washington, DC, 00-38.
Corbett, C. J., P. R. Kleindorfer. 2001a. Environmental management and operations management: Introduction to Part 1 (Manufacturing and Eco-Logistics). Production and Operations Management 10(2) 107-111.

Corbett, C. J., P. R. Kleindorfer. 2001b. Environmental management and operations: Introduction to Part 2 (Integrating Operations and Environmental Management Systems). Production and $\mathrm{Op}$ erations Management 10(3) 225-227.

Cramton, P., S. Kerr. 2002. Tradeable carbon permit auctions-How and why to auction not grandfather. Energy Policy 30 333-345.

Daripa, A. 2001. A theory of treasury auctions. Journal of International Money and Finance 20 743-767.

Debo, L. G., L. B. Toktay, L. N. Van Wassenhove. 2005. Market segmentation and product technology selection for remanufacturable products. Management Science 51(8) 1193-1205.

Ellerman, A. D. 1998. Electric Utility Response to Allowances: From Autarkic to Market-Based Compliance. Working Paper, MIT Center for Energy and Environmental Policy Research, 98-009.

Environmental Protection Agency, United States. 2005. Acid Rain Program: 2004 Progress Report.

Ferguson, M. E., L. B. Toktay. 2006. The effect of competition on 
recovery strategies. Production and Operations Management 15(3) 351-368.

Fischer, C., I. W. H. Parry, W. A. Pizer. 2003. Instrument choice for environmental protection when technological innovation is endogenous. Journal of Environmental Economics and Management $80249-267$.

Fullerton, D., G. Metcalf. 2001. Environmental controls, scarcity rents, and pre-existing distortions. Journal of Public Economics $\mathbf{8 0}$ 249-267.

Guide, V. D. R., Jr., L. N. Van Wassenhove, Eds. 2003. Business Aspects of Closed-Loop Supply Chains. Carnegie Mellon University Press, Pittsburgh, PA.

Guide, V. D. R., Jr., L. N. Van Wassenhove. 2006a. Closed-loop supply chains: An introduction to the feature issue (Part 1). Production and Operations Management 15(3) 345-350.

Guide, V. D. R., Jr., L. N. Van Wassenhove. 2006b. Closed-loop supply chains: An introduction to the feature issue (Part 2) Production and Operations Management 15(4) 471-472.

Hortaçsu, A. 2002. Bidding Behavior in Divisible Good Auctions: Theory and Evidence from the Turkish Treasury Auction Market. Working Paper, Department of Economics, Stanford University.

Kennedy, P. W. 2002. Optimal early action on greenhouse gas emissions. Canadian Journal of Economics 35(1) 16-35.

Kleindorfer, P. R., K. Singhal, L. N. Van Wassenhove. 2005. Sustainable operations management. Production and Operations Management 14(4) 482-492.

Laffont, J., J. Tirole. 1996a. Pollution permits and compliance strategies. Journal of Public Economics 62 85-125.

Laffont, J., J. Tirole. 1996b. Pollution permits and environmental innovation. Journal of Public Economics 62 127-140.

Lyon, R. M. 1986. Equilibrium properties of auctions and alternative procedures for allocating transferable permits. Journal of Environmental Economics and Management 13(2) 129-152.

Lyon, T. P., J. W. Maxwell. 2004. Corporate Environmentalism and Public Policy. Cambridge University Press, Cambridge, UK.

Montero, J.-P. 2002. Permits, standards, and technology innovation. Journal of Environmental Economics and Management 44 23-44.

Montero, J.-P., A. D. Ellerman. 1998. Explaining Low Sulfur Dioxide Allowance Prices: The Effect of Expectation Errors and Irreversibility. Working Paper, MIT Center for Energy and Environmental Policy Research, 98-011.

Muller, A. R., S. Mestelman. 1998. What have we learned from emissions trading experiments? Managerial and Decision Economics 19 225-238.
National Round Table on the Environment and the Economy (NRTEE), Canada. 2002. The ABCs of Emissions Trading: An Overview. Ontario, Canada.

Parry, I. W. H., M. Toman. 2002. Early emission reduction programs: An application to $\mathrm{CO}_{2}$ policy. The Energy Journal 23(1) 73-95.

Porter, M. E., C. van der Linde. Green and competitive: Ending the stalemate. 1995. Harvard Business Review (Sep-Oct) 120-134.

Rajaram, K., C. J. Corbett. 2002. Achieving environmental and productivity improvements through model-based process redesign. Operations Research 50(5) 751-763.

Savaskan, R. C., S. Bhattacharya, L. N. Van Wassenhove. 2004. Closed-loop supply chain models with product remanufacturing. Management Science 50(2) 239-252.

Schmalensee, R., P. L. Joskow, A. D. Ellerman, J.-P. Montero, E. M. Bailey. 1998. An interim evaluation of sulfur dioxide emissions trading. Journal of Economic Perspectives 12(3) 53-68.

Smith, A. E., M. T. Ross, W. D. Montgomery. 2002. Implications of Trading Implementation Design for Equity-Efficiency Trade-offs in Carbon Permit Allocations. Working Paper, Charles River Associates, Washington, DC.

Stavins, R. N. 1998. What can we learn from the grand policy experiment? Lessons from $\mathrm{SO}_{2}$ allowance trading. Journal of Economic Perspectives 12(3) 69-88.

Stuart, J. A., J. C. Ammons, L. J. Turbini. 1999. A product and process selection model with multidisciplinary environmental considerations. Operations Research 47(2) 221-234.

Sunnevåg, K. J. 2003. Auction design for the allocation of emission permits in the presence of market power. Environmental and Resource Economics 26(3) 384-400.

Tietenberg, T. H. 2001. Emissions Trading Programs. Ashgate Publishing Ltd., Aldershot, UK.

Unold, W., T. Requate. 2001. Pollution control by options trading. Economics Letters 73 353-358.

Wall Street Journal, The. 2005a. EPA Sets Strict Limits on Power-Plant Emissions. (Mar 11).

Wall Street Journal, The. 2005b. Utilities Start to Feel Bite of $\mathrm{SO}_{2}$ Caps. (Apr 13).

Wall Street Journal, The. 2005c. States Divide over Greenhouse Gases: Northeast and West Coast Clusters Feud over Implementing Limits on Emissions. (Dec 13).

Wall Street Journal, The. 2006. U.K., California to Weigh Market for Reducing Greenhouse Gases. (Aug 1).

Wilson, R. 1979. Auctions of shares. The Quarterly Journal of Economics 93(4) 675-689. 